\title{
HOMOTOPIC PROPERTIES OF KÄHLER ORBIFOLDS
}

\author{
GIOVANNI BAZZONI, INDRANIL BISWAS, MARISA FERNÁNDEZ, VICENTE MUÑOZ, \\ AND ALEKSY TRALLE
}

To Simon Salamon on the occasion of his 60th birthday

\begin{abstract}
We prove the formality and the evenness of odd-degree Betti numbers for compact Kähler orbifolds, by adapting the classical proofs for Kähler manifolds. As a consequence, we obtain examples of symplectic orbifolds not admitting any Kähler orbifold structure. We also review the known examples of non-formal simply connected Sasakian manifolds, and produce an example of a non-formal quasi-regular Sasakian manifold with Betti numbers $b_{1}=0$ and $b_{2}>1$.
\end{abstract}

\section{INTRODUCTION}

A Kähler manifold $M$ is a complex manifold, admitting a Hermitian metric $h$, such that the $(1,1)$-form $\omega=\operatorname{Im} h$ is closed, and so symplectic, where $\operatorname{Im} h$ is the imaginary part of $h$. The real part $g=\operatorname{Re} h$ of $h$ is a Riemannian metric which is is called the Kähler metric associated to $\omega$. If a compact manifold admits a Kähler metric, then it inherits some very striking topological properties, for example: theory of Kähler groups, evenness of odd-degree Betti numbers, hard Lefschetz theorem, formality of the rational homotopy type (see [11, 43]).

Kähler metrics can be also defined on orbifolds. A smooth orbifold $X$, of dimension $n$, is a Hausdorff topological space admitting an open cover $\left\{U_{i}\right\}_{i \in I}$, such that each $U_{i}$ is homeomorphic to a quotient $\Gamma_{i} \backslash \widetilde{U}_{i}$, where $\widetilde{U}_{i} \subset \mathbb{R}^{n}$ is an open subset, $\Gamma_{i} \subset \operatorname{GL}(n, \mathbb{R})$ a finite group acting on $\widetilde{U}_{i}$, and there is a $\Gamma_{i}$-invariant continuous map $\varphi_{i}: \widetilde{U}_{i} \longrightarrow U_{i}$ inducing a homeomorphism from $\Gamma_{i} \backslash \widetilde{U}_{i}$ onto $U_{i}$. Moreover, the gluing maps are required to be smooth and compatible with the group action (see Section 3 for the details).

The orbifold differential forms on a smooth orbifold are defined in local charts as $\Gamma_{i^{-}}$ invariant differential forms on each open set $\widetilde{U}_{i}$, which are compatible with the gluing maps. The de Rham complex is defined in the same way as for smooth manifolds, and the de Rham cohomology is equal to the singular cohomology. This result and Poincaré duality theorem were first proved by Satake, who introduced the notion of orbifold under the name " $V$-manifold" 35]. Since Satake, various index theorems were generalized by Kawasaki to the category of $V$-manifolds (see [21, 22, 23] and the book by Atiyah [2]). In the late 1970s, Thurston [38] rediscovered the concept of $V$-manifold, under the name of orbifold, in his study of the geometry of 3-manifolds, and defined the orbifold fundamental group. Even though orbifolds were already very important objects in mathematics, with the work of Dixon, Harvey, Vafa and Witten on conformal field theory [12], the interest

2010 Mathematics Subject Classification. 57R18, 55S30.

Key words and phrases. Kähler orbifolds, Sasakian manifolds, symplectic orbifolds, formality, Massey products, hard Lefschetz theorem. 
on orbifolds dramatically increased, due to their role in string theory (see [1] and the references therein).

A complex orbifold, of complex dimension $n$, is an orbifold $X$ with charts $\left(U_{i}, \widetilde{U}_{i}, \Gamma_{i}, \varphi_{i}\right)$ as above satisfying the conditions that $\widetilde{U}_{i} \subset \mathbb{C}^{n}, \Gamma_{i} \subset \operatorname{GL}(n, \mathbb{C})$, and all the gluing maps are given by biholomorphisms. One can also define orbifold complex forms and orbifold Hermitian metrics on $X$ (see Section 5 for the details). A complex orbifold $X$ is said to be Kähler if $X$ admits an orbifold Hermitian metric such that the associated orbifold Kähler form is closed. The notion of complex orbifold was introduced, under the name of complex $V$-manifold, by Baily [3] who generalized the Hodge decomposition theorem to Riemannian $V$-manifolds.

Although compact Kähler orbifolds are not smooth manifolds in general, they continue to possess some topological properties of Kähler manifolds. There are two possible points of view to look at topological properties of orbifolds. One is to look at the topological properties of the underlying topological space, and the other is to look at specific orbifold invariants such as the orbifold fundamental group or the orbifold cohomology. We shall focus on the former, since the latter is more adequate for the interplay between the topological space and the subspaces defining the orbifold ramification locus. So when we talk of the fundamental group or the homology or cohomology of the orbifold, we refer to those of the underlying topological spaces.

A compact Kähler orbifold is the leaf space of a foliation on a compact manifold $Y$ [18, Proposition 4.1], and such a foliation is transversely Kähler [42, Proposition 1.4]. Moreover, the basic cohomology of $Y$ is isomorphic to the singular cohomology of the orbifold over $\mathbb{C}[34,5.3]$. In [42] it is proved that any compact Kähler orbifold satisfies the hard Lefschetz property. This is done by using a result of El Kacimi-Alaoui [13] which says that the basic cohomology of a transversely Kähler foliation on a compact manifold satisfies the hard Lefschetz property. On the other hand, the $d d^{c}$-lemma for the algebra of the basic forms of a transversely Kähler foliation was shown in [10]. Also in [13] it is proved that the basic Dolbeault cohomology of a transversely Kähler foliation on a compact manifold has the same properties as the Dolbeault cohomology of a compact Kähler manifold. So, compact Kähler orbifolds possess the earlier mentioned topological properties of Kähler manifolds. Regarding the fundamental group of a Kähler orbifold, the fundamental group of the topological space underlying the orbifold actually coincides with the fundamental group of a resolution [25, Theorem 7.8.1]. Therefore, these fundamental groups of Kähler orbifolds satisfy the same restrictions as the fundamental groups of compact Kähler manifolds.

The main purpose of this paper is to prove that compact Kähler orbifolds are formal. This is achieved by adapting the proof of formality for Kähler manifolds given in [11]. The machinery used is described in Sections 2, 3 and 4. In Sections 2 and 3 we focus on the formality of smooth manifolds and orbifolds, respectively, and in Section 4 we study elliptic operators on complex orbifolds following [43], but it was first developed by Baily in the aforementioned paper [3]. Then, in Section 5 the orbifold Dolbeault cohomology of a complex orbifold is defined, and the $\partial \bar{\partial}$-lemma for compact Kähler orbifolds is proved (Lemma 5.4). The formality of compact Kähler orbifolds is deduced using this (Theorem [5.5). Moreover, in Proposition 5.2 we prove that the orbifold Dolbeault cohomology is equipped with an analogue of the Hodge decomposition for Kähler manifolds. 
Consequently, the odd Betti numbers of compact Kähler orbifolds are even. (A Hodge decomposition theory for nearly Kähler manifolds was developed by Verbitsky in [41, where it is noted that this theory works also for nearly Kähler orbifolds.) In Section 6, we produce examples of symplectic orbifolds which do not admit any Kähler orbifold metric (as they are non-formal or they do not possess the hard Lefschetz property).

Closely related to Kähler orbifolds are Sasakian manifolds. Such a manifold is a Riemannian manifold $(N, g)$, of dimension $2 n+1$, such that its cone $\left(N \times \mathbb{R}^{+}, g^{c}=t^{2} g+d t^{2}\right)$ is Kähler, and so the holonomy group for $g^{c}$ is a subgroup of $\mathrm{U}(n+1)$. The Kähler structure on the cone induces a Sasakian structure on the base of the cone. In particular, the complex structure on the cone gives rise to a Reeb vector field.

If $N$ admits a Sasakian structure, then in [33] it is proved that $N$ also admits a quasiregular Sasakian structure. The space $X$ of leaves of a quasi-regular Sasakian structure is a Kähler orbifold with cyclic quotient singularities, and there is an orbifold circle bundle $S^{1} \hookrightarrow N \stackrel{\pi}{\longrightarrow} X$ such that the contact form $\eta$ satisfies the equation $d \eta=\pi^{*} \omega$, where $\omega$ is the orbifold Kähler form. If $X$ is a Kähler manifold, then the Sasakian structure on $N$ is regular.

However, opposed to Kähler orbifolds, formality is not an obstruction to the existence of a Sasakian structure even on simply connected manifolds [6]. On the other hand, all quadruple and higher order Massey products are trivial on any Sasakian manifold. In fact, in [6] it is proved that, for any $n \geq 3$, there exists a simply connected compact regular Sasakian manifold, of dimension $2 n+1$, which is non-formal, in fact not 3 -formal, in the sense of Definition 2.2. (Note that simply connected compact manifolds of dimension at most 6 are formal [16, 31].) In Section 7 we review these examples and show that they have a non-trivial (triple) Massey product, which implies that they are not formal.

Regarding the simply connected compact regular Sasakian manifolds that are formal, the odd-dimensional sphere $S^{2 n+1}$ is the most basic example of them. By Theorem 2.3 we know that any 7-dimensional simply connected compact manifold (Sasakian or not) with $b_{2} \leq 1$ is formal. In [15], examples are given of simply connected formal compact regular Sasakian manifolds, of dimension 7 , with second Betti number $b_{2} \geq 2$. This result and Proposition 7.1 (Section 7 ) show that, for every $n \geq 3$, there exists a simply connected compact regular Sasakian manifold, of dimension $2 n+1 \geq 7$, which is formal and has $b_{2} \neq 0$. We end up with an example of a quasi-regular (non-regular) Sasakian manifold with $b_{1}=0$ which is non-formal.

\section{Formality OF MANifolds}

In this section some definitions and results about minimal models and Massey products on smooth manifolds are reviewed; see [11, 14] for more details.

We work with the differential graded commutative algebras, or DGAs, over the field $\mathbb{R}$ of real numbers. The degree of an element $a$ of a DGA is denoted by $|a|$. $\operatorname{A~DGA~}(\mathcal{A}, d)$ is minimal if:

(1) $\mathcal{A}$ is free as an algebra, that is $\mathcal{A}$ is the free algebra $\bigwedge V$ over a graded vector space $V=\bigoplus_{i} V^{i}$, and 
(2) there is a collection of generators $\left\{a_{\tau}\right\}_{\tau \in I}$ indexed by some well ordered set $I$, such that $\left|a_{\mu}\right| \leq\left|a_{\tau}\right|$ if $\mu<\tau$ and each $d a_{\tau}$ is expressed in terms of the previous $a_{\mu}$, $\mu<\tau$. This implies that $d a_{\tau}$ does not have a linear part.

In our context, the main example of DGA is the de Rham complex $\left(\Omega^{*}(M), d\right)$ of a smooth manifold $M$, where $d$ is the exterior differential.

The cohomology of a differential graded commutative algebra $(\mathcal{A}, d)$ is denoted by $H^{*}(\mathcal{A}) . H^{*}(\mathcal{A})$ is naturally a DGA with the product inherited from that on $\mathcal{A}$ while the differential on $H^{*}(\mathcal{A})$ is identically zero.

A DGA $(\mathcal{A}, d)$ is called connected if $H^{0}(\mathcal{A})=\mathbb{R}$, and it is called 1-connected if, in addition, $H^{1}(\mathcal{A})=0$.

Morphisms between DGAs are required to preserve the degree and to commute with the differential. We shall say that $(\bigwedge V, d)$ is a minimal model of a differential graded commutative algebra $(\mathcal{A}, d)$ if $(\bigwedge V, d)$ is minimal and there exists a morphism of differential graded algebras

$$
\rho:(\bigwedge V, d) \longrightarrow(\mathcal{A}, d)
$$

inducing an isomorphism $\rho^{*}: H^{*}(\bigwedge V) \stackrel{\sim}{\longrightarrow} H^{*}(\mathcal{A})$ of cohomologies. In [20], Halperin proved that any connected differential graded algebra $(\mathcal{A}, d)$ has a minimal model unique up to isomorphism. For 1-connected differential algebras, a similar result was proved by Deligne, Griffiths, Morgan and Sullivan [11, 19, 37].

A minimal model of a connected smooth manifold $M$ is a minimal model $(\bigwedge V, d)$ for the de Rham complex $\left(\Omega^{*}(M), d\right)$ of differential forms on $M$. If $M$ is a simply connected manifold, then the dual of the real homotopy vector space $\pi_{i}(M) \otimes \mathbb{R}$ is isomorphic to the space $V^{i}$ of generators in degree $i$, for any $i$. The latter also happens when $i>1$ and $M$ is nilpotent, that is, the fundamental group $\pi_{1}(M)$ is nilpotent and its action on $\pi_{j}(M)$ is nilpotent for all $j>1$ (see [11]).

We say that a DGA $(\mathcal{A}, d)$ is a model of a manifold $M$ if $(\mathcal{A}, d)$ and $M$ have the same minimal model. Thus, if $(\bigwedge V, d)$ is the minimal model of $M$, we have

$$
(\mathcal{A}, d) \stackrel{\nu}{\longleftarrow}(\bigwedge V, d) \stackrel{\rho}{\longrightarrow}\left(\Omega^{*}(M), d\right)
$$

where $\rho$ and $\nu$ are quasi-isomorphisms, meaning morphisms of DGAs such that the induced homomorphisms in cohomology are isomorphisms.

Recall that a minimal algebra $(\bigwedge V, d)$ is called formal if there exists a morphism of differential algebras $\psi:(\bigwedge V, d) \longrightarrow\left(H^{*}(\bigwedge V), 0\right)$ inducing the identity map on cohomology. A DGA $(\mathcal{A}, d)$ is formal if its minimal model is formal.

A smooth manifold $M$ is called formal if its minimal model is formal. Many examples of formal manifolds are known: spheres, projective spaces, compact Lie groups, symmetric spaces, flag manifolds, and compact Kähler manifolds.

The formality property of a minimal algebra is characterized as follows.

Proposition 2.1 ([11]). A minimal algebra $(\bigwedge V, d)$ is formal if and only if the space $V$ can be decomposed into a direct sum $V=C \oplus N$ with $d(C)=0$ and d injective on $N$, such that every closed element in the ideal $I(N)$ in $\bigwedge V$ generated by $N$ is exact. 
This characterization of formality can be weakened using the concept of $s$-formality introduced in [16].

Definition 2.2. A minimal algebra $(\bigwedge V, d)$ is $s$-formal $(s>0)$ if for each $i \leq s$ the space $V^{i}$ of generators of degree $i$ decomposes as a direct sum $V^{i}=C^{i} \oplus N^{i}$, where the spaces $C^{i}$ and $N^{i}$ satisfy the following conditions:

(1) $d\left(C^{i}\right)=0$,

(2) the differential map $d: N^{i} \longrightarrow \bigwedge V$ is injective, and

(3) any closed element in the ideal $I_{s}=I\left(\bigoplus_{i \leq s} N^{i}\right)$, generated by the space $\bigoplus_{i \leq s} N^{i}$ in the free algebra $\bigwedge\left(\bigoplus_{i \leq s} V^{i}\right)$, is exact in $\bigwedge V$.

A smooth manifold $M$ is $s$-formal if its minimal model is $s$-formal. Clearly, if $M$ is formal then $M$ is $s$-formal for every $s>0$. The main result of [16] shows that sometimes the weaker condition of $s$-formality implies formality.

Theorem 2.3 ([16]). Let $M$ be a connected and orientable compact differentiable manifold of dimension $2 n$ or $(2 n-1)$. Then $M$ is formal if and only if it is $(n-1)$-formal.

One can check that any simply connected compact manifold is 2-formal. Therefore, Theorem 2.3 implies that any simply connected compact manifold of dimension at most six is formal. (This result was proved earlier in [31].)

In order to detect non-formality, instead of computing the minimal model, which is usually a lengthy process, one can use Massey products, which are obstructions to formality. The simplest type of Massey product is the triple (also known as ordinary) Massey product. This will be defined next.

Let $(\mathcal{A}, d)$ be a DGA (in particular, it can be the de Rham complex of differential forms on a smooth manifold). Suppose that there are cohomology classes $\left[a_{i}\right] \in H^{p_{i}}(\mathcal{A})$, $p_{i}>0,1 \leq i \leq 3$, such that $a_{1} \cdot a_{2}$ and $a_{2} \cdot a_{3}$ are exact. Write $a_{1} \cdot a_{2}=d a_{1,2}$ and $a_{2} \cdot a_{3}=d a_{2,3}$. The (triple) Massey product of the classes $\left[a_{i}\right]$ is defined as

$$
\left\langle\left[a_{1}\right],\left[a_{2}\right],\left[a_{3}\right]\right\rangle=\left[a_{1} \cdot a_{2,3}+(-1)^{p_{1}+1} a_{1,2} \cdot a_{3}\right] \in \frac{H^{p_{1}+p_{2}+p_{3}-1}(\mathcal{A})}{\left[a_{1}\right] \cdot H^{p_{2}+p_{3}-1}(\mathcal{A})+\left[a_{3}\right] \cdot H^{p_{1}+p_{2}-1}(\mathcal{A})} .
$$

Note that a Massey product $\left\langle\left[a_{1}\right],\left[a_{2}\right],\left[a_{3}\right]\right\rangle$ on $\left(\mathcal{A}, d_{\mathcal{A}}\right)$ is zero (or trivial) if and only if there exist $\widetilde{x}, \widetilde{y} \in \mathcal{A}$ such that $a_{1} \cdot a_{2}=d_{\mathcal{A}} \widetilde{x}, a_{2} \cdot a_{3}=d_{\mathcal{A}} \widetilde{y}$ and

$$
0=\left[a_{1} \cdot \widetilde{y}+(-1)^{p_{1}+1} \widetilde{x} \cdot a_{3}\right] \in H^{p_{1}+p_{2}+p_{3}-1}(\mathcal{A}) .
$$

We will use also the following property.

Lemma 2.4. Let $M$ be a connected smooth manifold. Then, Massey products on $M$ can be calculated by using any model of $M$.

Proof. It is enough to prove the following: $\varphi:\left(\mathcal{A}, d_{\mathcal{A}}\right) \longrightarrow\left(\mathcal{B}, d_{\mathcal{B}}\right)$ is a quasi-isomorphism, then

$$
\varphi^{*}\left(\left\langle\left[a_{1}\right],\left[a_{2}\right],\left[a_{3}\right]\right\rangle\right)=\left\langle\left[a_{1}^{\prime}\right],\left[a_{2}^{\prime}\right],\left[a_{3}^{\prime}\right]\right\rangle
$$


for $\left[a_{j}^{\prime}\right]=\varphi^{*}\left(\left[a_{j}\right]\right)$. But this is clear; indeed, take $a_{1} \cdot a_{2}=d_{\mathcal{A}} x, a_{2} \cdot a_{3}=d_{\mathcal{A}} y$ and let

$$
f=\left[a_{1} \cdot y+(-1)^{p_{1}+1} x \cdot a_{3}\right] \in \frac{H^{p_{1}+p_{2}+p_{3}-1}(\mathcal{A})}{\left[a_{1}\right] \cdot H^{p_{2}+p_{3}-1}(\mathcal{A})+\left[a_{3}\right] \cdot H^{p_{1}+p_{2}-1}(\mathcal{A})}
$$

be its Massey product $\left\langle\left[a_{1}\right],\left[a_{2}\right],\left[a_{3}\right]\right\rangle$. Then the elements $a_{j}^{\prime}=\varphi\left(a_{j}\right)$ satisfy $a_{1}^{\prime} \cdot a_{2}^{\prime}=d_{\mathcal{B}} x^{\prime}$, $a_{2}^{\prime} \cdot a_{3}^{\prime}=d_{\mathcal{B}} y^{\prime}$, where $x^{\prime}=\varphi(x), y^{\prime}=\varphi(y)$. Therefore,

$$
f^{\prime}=\left[a_{1}^{\prime} \cdot y^{\prime}+(-1)^{p_{1}+1} x^{\prime} \cdot a_{3}^{\prime}\right]=\varphi^{*}(f) \in \frac{H^{p_{1}+p_{2}+p_{3}-1}(\mathcal{B})}{\left[a_{1}^{\prime}\right] \cdot H^{p_{2}+p_{3}-1}(\mathcal{B})+\left[a_{3}^{\prime}\right] \cdot H^{p_{1}+p_{2}-1}(\mathcal{B})}
$$

is the Massey product $\left\langle\left[a_{1}^{\prime}\right],\left[a_{2}^{\prime}\right],\left[a_{3}^{\prime}\right]\right\rangle$.

Now we move to the definition of higher Massey products (see [40]). Given

$$
\left[a_{i}\right] \in H^{*}(\mathcal{A}), \quad 1 \leq i \leq t, \quad t \geq 3,
$$

the Massey product $\left\langle\left[a_{1}\right],\left[a_{2}\right], \cdots,\left[a_{t}\right]\right\rangle$, is defined if there are elements $a_{i, j}$ on $\mathcal{A}$, with $1 \leq i \leq j \leq t$ and $(i, j) \neq(1, t)$, such that

$$
\begin{aligned}
a_{i, i} & =a_{i} \\
d a_{i, j} & =\sum_{k=i}^{j-1}(-1)^{\left|a_{i, k}\right|} a_{i, k} \cdot a_{k+1, j} .
\end{aligned}
$$

Then the Massey product is the set of all cohomology classes

$$
\begin{gathered}
\left\langle\left[a_{1}\right],\left[a_{2}\right], \cdots,\left[a_{t}\right]\right\rangle \\
=\left\{\left[\sum_{k=1}^{t-1}(-1)^{\left|a_{1, k}\right|} a_{1, k} \cdot a_{k+1, t}\right] \mid a_{i, j} \text { as in (2.1) }\right\} \subset H^{\left|a_{1}\right|+\cdots+\left|a_{t}\right|-(t-2)}(\mathcal{A}) .
\end{gathered}
$$

We say that the Massey product is zero if

$$
0 \in\left\langle\left[a_{1}\right],\left[a_{2}\right], \cdots,\left[a_{t}\right]\right\rangle .
$$

Note that the higher order Massey product $\left\langle\left[a_{1}\right],\left[a_{2}\right], \cdots,\left[a_{t}\right]\right\rangle$ of order $t \geq 4$ is defined if all the Massey products $\left\langle\left[a_{i}\right], \cdots,\left[a_{i+p-1}\right]\right\rangle$ of order $p$, where $3 \leq p \leq t-1$ and $1 \leq i \leq$ $t-p+1$, are defined and trivial.

Massey products are related to formality by the following well-known result.

Theorem 2.5 ([11, 40]). A DGA which has a non-zero Massey product is not formal.

Another obstruction to the formality is given by the $a$-Massey products introduced in [9], which generalize the triple Massey product and have the advantage of being simpler to compute compared to the higher order Massey products. They are defined as follows. Let $(\mathcal{A}, d)$ be a DGA, and let $a, b_{1}, \ldots, b_{n} \in \mathcal{A}$ be closed elements such that the degree $|a|$ of $a$ is even and $a \cdot b_{i}$ is exact for all $i$. Let $\xi_{i}$ be any form such that $d \xi_{i}=a \cdot b_{i}$. Then the $n^{\text {th }}$ order a-Massey product of the $b_{i}$ is the subset

$$
:=\left\{\left[\sum_{i}(-1)^{\left|\xi_{1}\right|+\cdots+\left|\xi_{i-1}\right|} \xi_{1} \cdot \ldots \cdot \xi_{i-1} \cdot b_{i} \cdot \xi_{i+1} \cdot \ldots \cdot \xi_{n}\right] \mid d \xi_{i}=a \cdot b_{i}\right\} \subset H^{*}(\mathcal{A}) .
$$

We say that the $a$-Massey product is zero if $0 \in\left\langle a ; b_{1}, \ldots, b_{n}\right\rangle$.

Theorem 2.6 ([9]). A DGA which has a non-zero a-Massey product is not formal. 


\section{ORBIFOLDS}

In this section, we collect some results about smooth orbifolds and formality of these spaces (see [1, 7, 19, 24, 35, 36, 38]).

Let $X$ be a topological space. Fix an integer $n>0$. An $\operatorname{orbifold} \operatorname{chart}(U, \widetilde{U}, \Gamma, \varphi)$ on $X$ consists of an open set $U \subset X$, a connected and open set $\widetilde{U} \subset \mathbb{R}^{n}$, a finite group $\Gamma \subset \operatorname{GL}(n, \mathbb{R})$ acting smoothly and effectively on $\widetilde{U}$, and a continuous map

$$
\varphi: \widetilde{U} \longrightarrow U
$$

which is $\Gamma$-invariant (that is $\varphi=\varphi \circ \gamma$, for all $\gamma \in \Gamma$ ) and such that it induces a homeomorphism

$$
\Gamma \backslash \widetilde{U} \stackrel{\cong}{\longrightarrow} U
$$

from the quotient space $\Gamma \backslash \widetilde{U}$ onto $U$.

Definition 3.1. A smooth orbifold $X$, of dimension $n$, is a Hausdorff, paracompact topological space endowed with an orbifold atlas $\mathcal{A}=\left\{\left(U_{i}, \widetilde{U}_{i}, \Gamma_{i}, \varphi_{i}\right)\right\}_{i \in I}$, that is $\mathcal{A}$ is a family of orbifold charts which satisfy the following conditions:

i) $\left\{U_{i}\right\}_{i \in I}$ is an open cover of $X$;

ii) If $\left(U_{i}, \widetilde{U}_{i}, \Gamma_{i}, \varphi_{i}\right)$ and $\left(U_{j}, \widetilde{U}_{j}, \Gamma_{j}, \varphi_{j}\right), i, j \in I$, are two orbifold charts, with $U_{i} \cap$ $U_{j} \neq \emptyset$, then for each point $p \in U_{i} \cap U_{j}$ there exists an orbifold chart $\left(U_{k}, \widetilde{U}_{k}, \Gamma_{k}, \varphi_{k}\right)$ $(k \in I)$ such that $p \in U_{k} \subset U_{i} \cap U_{j}$;

iii) If $\left(U_{i}, \widetilde{U}_{i}, \Gamma_{i}, \varphi_{i}\right)$ and $\left(U_{j}, \widetilde{U}_{j}, \Gamma_{j}, \varphi_{j}\right), i, j \in I$, are two orbifold charts, with $U_{i} \subset U_{j}$, then there exist a smooth embedding, called change of charts (or embedding or gluing map)

$$
\rho_{i j}: \widetilde{U}_{i} \longrightarrow \widetilde{U}_{j}
$$

(so that $\widetilde{U}_{i}$ and $\rho_{i j}\left(\widetilde{U}_{i}\right)$ are diffeomorphic) such that

$$
\varphi_{i}=\varphi_{j} \circ \rho_{i j}
$$

Note that, in most references, the definition given of orbifold chart $(U, \widetilde{U}, \Gamma, \varphi)$ does not explicitly require the condition that the finite group $\Gamma$ is such that $\Gamma \subset \operatorname{GL}(n, \mathbb{R})$. But since smooth actions are locally linearizable (see [8, page 308]), any orbifold has an atlas consisting of linear charts, that is charts of the form $\left(U_{i}, \mathbb{R}^{n}, \Gamma_{i}, \varphi_{i}\right)$ where $\Gamma_{i}$ acts on $\mathbb{R}^{n}$ via an orthogonal representation $\Gamma_{i} \subset \varnothing(n)$. Since $\Gamma_{i}$ is finite, we can consider an orbifold atlas on a topological space $X$ as given in Definition 3.1 .

As with smooth manifolds, two orbifold atlases $\mathcal{A}$ and $\mathcal{A}^{\prime}$ on $X$ are said to be equivalent if $\mathcal{A} \cup \mathcal{A}^{\prime}$ is also an orbifold atlas. Equivalent atlases on $X$ are regarded as defining the same orbifold structure on $X$. Every orbifold atlas for $X$ is contained in a unique maximal one, and two orbifold atlases are equivalent if and only if they are contained in the same maximal one.

Now, we consider some important points about Definition 3.1. Suppose that $X$ is a smooth orbifold, with two orbifold charts $\left(U_{i}, \widetilde{U}_{i}, \Gamma_{i}, \varphi_{i}\right)$ and $\left(U_{j}, \widetilde{U}_{j}, \Gamma_{j}, \varphi_{j}\right)$, such that $U_{i} \subset U_{j}$. Let $\rho_{i j}: \widetilde{U}_{i} \longrightarrow \widetilde{U}_{j}$ be a change of charts (in the sense of Definition 3.1). Note that $\rho_{i j} \circ \gamma: \widetilde{U}_{i} \longrightarrow \widetilde{U}_{j}$ is also a change of charts, for all $\gamma \in \Gamma_{i}$. We will see that, for $\gamma \in \Gamma_{i}$, there is an element $\widetilde{\gamma} \in \Gamma_{j}$ such that $\rho_{i j} \circ \gamma=\widetilde{\gamma} \circ \rho_{i j}$. In [28] it is proved the 
following result, which was proved by Satake in 35] under the added assumption that the fixed point set has codimension at least two.

Proposition 3.2. [28, Proposition A.1] Let $\left(U_{i}, \widetilde{U}_{i}, \Gamma_{i}, \varphi_{i}\right)$ and $\left(U_{j}, \widetilde{U}_{j}, \Gamma_{j}, \varphi_{j}\right)$ be two orbifold charts on $X$, with $U_{i} \subset U_{j}$. If $\rho_{i j}, \mu_{i j}: \widetilde{U}_{i} \longrightarrow \widetilde{U}_{j}$ are two change of charts, then there exists a unique $\gamma_{j} \in \Gamma_{j}$ such that $\mu_{i j}=\gamma_{j} \circ \rho_{i j}$.

As a consequence of Proposition 3.2 , a change of orbifold charts $\rho_{i j}: \widetilde{U}_{i} \longrightarrow \widetilde{U}_{j}$ induces an injective homomorphism $f_{i j}: \Gamma_{i} \longrightarrow \Gamma_{j}$ which is given by

$$
\rho_{i j} \circ \gamma=f_{i j}(\gamma) \circ \rho_{i j}
$$

that is $\rho_{i j}(\gamma \cdot x)=f_{i j}(\gamma) \cdot \rho_{i j}(x)$, for all $\gamma \in \Gamma_{i}$ and $x \in \widetilde{U}_{i}$.

Also in [28] it is proved the following.

Lemma 3.3. [28, Lemma A.2] Let $\left(U_{i}, \widetilde{U}_{i}, \Gamma_{i}, \varphi_{i}\right)$ and $\left(U_{j}, \widetilde{U}_{j}, \Gamma_{j}, \varphi_{j}\right)$ be two orbifold charts on $X$, with $U_{i} \subset U_{j}$. Consider $\rho_{i j}: \widetilde{U}_{i} \longrightarrow \widetilde{U}_{j}$ a change of charts which is equivariant with respect to the injective homomorphism $f_{i j}: \Gamma_{i} \longrightarrow \Gamma_{j}$. If there exists an element $\gamma_{j} \in \Gamma_{j}$ such that $\rho_{i j}\left(\widetilde{U}_{i}\right) \cap \gamma_{j} \cdot \rho_{i j}\left(\widetilde{U}_{i}\right) \neq \emptyset$, then $\gamma_{j} \in \operatorname{Im}\left(f_{i j}\right)$, and so $\rho_{i j}\left(\widetilde{U}_{i}\right)=\gamma_{j} \cdot \rho_{i j}\left(\widetilde{U}_{i}\right)$.

Let $X$ be a smooth orbifold, with an atlas $\left\{\left(U_{i}, \widetilde{U}_{i}, \Gamma_{i}, \varphi_{i}\right)\right\}$, and let $p \in X$. Consider $\left(U_{i}, \widetilde{U}_{i}, \Gamma_{i}, \varphi_{i}\right)$ an orbifold chart around $p$, that is $p=\varphi_{i}(x) \in U_{i}$ with $x \in \widetilde{U}_{i}$, and denote by $\Gamma_{i}(x) \subset \Gamma_{i}$ the isotropy subgroup for the point $x$. Note that, up to conjugation, the group $\Gamma_{i}(x)$ does not depend on the choice of the orbifold chart around $p$. In fact, if $\left(U_{i}, \widetilde{U}_{i}, \Gamma_{i}, \varphi_{i}\right)$ is an orbifold chart around $p$ and $p=\varphi_{i}(x)=\varphi_{i}\left(x^{\prime}\right) \in U_{i}$ with $x, x^{\prime} \in \widetilde{U}_{i}$, then $\Gamma_{i}\left(x^{\prime}\right)$ is conjugate to $\Gamma_{i}(x)$. (Indeed, there is a group isomorphism $L_{a}: \Gamma_{i}(x) \longrightarrow$ $\Gamma_{i}\left(x^{\prime}\right)$ such that, for $\gamma \in \Gamma_{i}(x), L_{a}(\gamma)=a \gamma a^{-1}$ with $a \in \operatorname{GL}(n, \mathbb{R})$.) Moreover, if $\left(U_{j}, \widetilde{U}_{j}, \Gamma_{j}, \varphi_{j}\right)$ is other orbifold chart with $p=\varphi_{j}(y) \in U_{j}$, then we have a third orbifold chart $\left(U_{k}, \widetilde{U}_{k}, \Gamma_{k}, \varphi_{k}\right)$ around $p=\varphi_{k}(z) \in U_{k}$, together with smooth embeddings

$$
\rho_{k i}: \widetilde{U}_{k} \longrightarrow \widetilde{U}_{i}, \quad \rho_{k j}: \widetilde{U}_{k} \longrightarrow \widetilde{U}_{j},
$$

and injective homomorphisms $f_{k i}: \Gamma_{k} \longrightarrow \Gamma_{i}, f_{k j}: \Gamma_{k} \longrightarrow \Gamma_{j}$ such that $\rho_{k i}$ and $\rho_{k j}$ satisfy (3.1) with respect to $f_{k i}$ and $f_{k j}$, respectively. Thus, $f_{k i}$ and $f_{k j}$ define monomorphisms $\Gamma_{k}(z) \hookrightarrow \Gamma_{i}(x)$ and $\Gamma_{k}(z) \hookrightarrow \Gamma_{j}(y)$. But these monomorphisms must be also onto by Lemma 3.3. So,

$$
\Gamma_{k}(z) \cong \Gamma_{j}(y) \cong \Gamma_{i}(x) .
$$

This justifies that the group $\Gamma_{i}(x)$ is called the (local) isotropy group of $p$, and it is denoted $\Gamma_{p}$. When $\Gamma_{p} \neq \mathrm{Id}$, the point $p$ is said to be a singular point of the orbifold $X$. The points $p$ with $\Gamma_{p}=$ Id are called regular points. The set of singular points

$$
S=\left\{p \in X \mid \Gamma_{p} \neq \mathrm{Id}\right\}
$$

is called the singular locus of the orbifold $X$ (or orbifold singular set). Then $X-S$ is a smooth $n$-dimensional manifold.

The singular locus can be stratified according to the isotropy groups. For each group $H$, we have a subset $S_{H}=\left\{p \in X \mid \Gamma_{p}=H\right\}$. It is easily seen that the connected components of $S_{H}$ are locally closed smooth submanifolds of $X$. Moreover, the closure $\bar{S}_{H}$ contains components of other $S_{H^{\prime}}$, with $H<H^{\prime}$. This is an immediate consequence of the fact 
that it holds on every orbifold chart (in an orbifold chart $(U, \widetilde{U}, \Gamma, \varphi)$, the sets $S_{H}$ are linear subsets of $\widetilde{U})$. As a consequence, we can give a CW-structure to $X$ compatible with the stratification, that is, such that the subsets $\bar{S}_{H}$ are CW-subcomplexes.

Any smooth manifold is a smooth orbifold for which each of the finite groups $\Gamma_{i}$ is the trivial group, so that we get $\widetilde{U}_{i}$ homeomorphic to $U_{i}$. The most natural examples of orbifolds appear when we take the quotient space $X=M / \Gamma$ of a smooth manifold $M$ by a finite group $\Gamma$ acting smoothly and effectively on $M$. Let $\pi: M \rightarrow X$ be the natural projection. Note that given un point $p \in M$ with isotropy group $\Gamma_{p} \subset \Gamma$, then there is a chart $(U, \widetilde{U}, \phi)$ of $p=\phi(x) \in U$ in $M$, with $U=\phi(\widetilde{U})$, such that $U$ is $\Gamma_{p}$-invariant. Then, an orbifold chart of $\pi(p) \in X$ is $\left(\pi(U), \widetilde{U}, \Gamma_{p}, \pi \circ \phi\right)$, the change of charts $\rho_{i j}$ are the change of coordinates on the manifold $M$, and the monomorphisms $f_{i j}$ are the identity map of $\Gamma_{p}$. Such an orbifold

$$
X=M / \Gamma
$$

is called effective global quotient orbifold [1, Definition 1.8].

Moreover, if $M$ is oriented and the action of $\Gamma$ preserves the orientation, then $X$ is an oriented orbifold. In general, an orbifold $X$, with atlas $\left\{\left(U_{i}, \widetilde{U}_{i}, \Gamma_{i}, \varphi_{i}\right)\right\}$, is oriented if each $\widetilde{U}_{i}$ is oriented, the action of $\Gamma_{i}$ is orientation-preserving, and all the change of charts $\rho_{i j}: \widetilde{U}_{i} \longrightarrow \widetilde{U}_{j}$ are orientation-preserving.

Definition 3.4 ([7]). Let $X$ and $Y$ be two orbifolds (not necessarily of the same dimension) and let $\left\{\left(U_{i}, \widetilde{U}_{i}, \Gamma_{i}, \varphi_{i}\right)\right\}$ and $\left\{\left(V_{j}, \widetilde{V}_{j}, \Upsilon_{j}, \psi_{j}\right)\right\}$ be atlases for $X$ and $Y$, respectively. A map $f: X \longrightarrow Y$ is said to be an orbifold map (or smooth map) if $f$ is a continuous map between the underlying topological spaces, and for every point $p \in X$ there are orbifold charts $\left(U_{i}, \widetilde{U}_{i}, \Gamma_{i}, \varphi_{i}\right)$ and $\left(V_{i}, \widetilde{V}_{i}, \Upsilon_{i}, \psi_{i}\right)$ for $p$ and $f(p)$, respectively, with $f\left(U_{i}\right) \subset V_{i}$, a differentiable map $\widetilde{f}_{i}: \widetilde{U}_{i} \longrightarrow \widetilde{V}_{i}$, and a homomorphism $\varpi_{i}: \Gamma_{i} \rightarrow \Upsilon_{i}$ such that $\tilde{f}_{i} \circ \gamma=\varpi_{i}(\gamma) \circ \widetilde{f}_{i}$ for all $\gamma \in \Gamma_{i}$, and

$$
f_{\mid U_{i}} \circ \varphi_{i}=\psi_{i} \circ \tilde{f}_{i}
$$

Moreover, $f$ is said to be good if every map $\tilde{f}_{i}$ is compatible with the changes of charts in the following sense:

i) if $\rho_{i j}: \widetilde{U}_{i} \longrightarrow \widetilde{U}_{j}$ is a change of charts for $p$, then there is a change of charts $\mu\left(\rho_{i j}\right): \widetilde{V}_{i} \longrightarrow \widetilde{V}_{j}$ for $f(p)$ such that

$$
\tilde{f}_{j} \circ \rho_{i j}=\mu\left(\rho_{i j}\right) \circ \tilde{f}_{i} ;
$$

ii) if $\rho_{k i}: \widetilde{U}_{k} \longrightarrow \widetilde{U}_{i}$ is a change of charts for $p$, then

$$
\mu\left(\rho_{i j} \circ \rho_{k i}\right)=\mu\left(\rho_{i j}\right) \circ \mu\left(\rho_{k i}\right) .
$$

Therefore, an orbifold map $f: X \longrightarrow Y$ is determined by a smooth map $\widetilde{f}_{i}: \widetilde{U}_{i} \longrightarrow \widetilde{V}_{i}$, for every orbifold chart $\left(U_{i}, \widetilde{U}_{i}, \Gamma_{i}, \varphi_{i}\right)$ on $X$, such that every $\widetilde{f}_{i}$ is $\Gamma_{i}$-equivariant and compatible with the change of orbifold charts.

Note that conditions i) and ii) in Definition 3.4 imply that the composition of orbifold maps is an orbifold map. Moreover, if $f: X \longrightarrow Y$ is an orbifold map, then there exists an induced homomorphism from $\Gamma_{p}$ to $\Upsilon_{f(p)}$. Also, considering $\mathbb{R}$ as an orbifold, we can define orbifold functions on an orbifold $X$ as orbifold maps $f: X \longrightarrow \mathbb{R}$. 
Two orbifolds $X$ and $Y$ are said to be diffeomorphic if there exist orbifold maps $f: X \longrightarrow Y$ and $g: Y \longrightarrow X$ such that $g \circ f=1_{X}$ and $f \circ g=1_{Y}$, where $1_{X}$ and $1_{Y}$ are the respective identity maps. Note that a diffeomorphism between orbifolds gives a homeomorphism between the underlying topological spaces.

Many of the usual differential geometric concepts that hold for smooth manifolds also hold for smooth orbifolds; in particular, the notion of vector bundle [7, Definition 4.2.7]. Using transition maps, orbifold vector bundles can be defined as follows [36].

Definition 3.5. Let $X$ be a smooth orbifold, of dimension $n$, and let $\left\{\left(U_{i}, \widetilde{U}_{i}, \Gamma_{i}, \varphi_{i}\right)\right\}_{i \in I}$ be an atlas on $X$. An orbifold vector bundle over $X$ and fiber $\mathbb{R}^{m}$ consists of a smooth orbifold $E$, of dimension $m+n$, and an orbifold map

$$
\pi: E \longrightarrow X
$$

called projection, satisfying the following conditions:

i) For every orbifold chart $\left(U_{i}, \widetilde{U}_{i}, \Gamma_{i}, \varphi_{i}\right)$ on $X$, there exists a homomorphism

$$
\rho_{i}: \Gamma_{i} \longrightarrow \mathrm{GL}\left(\mathbb{R}^{m}\right)
$$

and an orbifold chart $\left(V_{i}, \widetilde{V}_{i}, \Gamma_{i}, \Psi_{i}\right)$ on $E$, such that $V_{i}=\pi^{-1}\left(U_{i}\right), \widetilde{V}_{i}=\widetilde{U}_{i} \times \mathbb{R}^{m}$, the action of $\Gamma_{i}$ on $\widetilde{U}_{i} \times \mathbb{R}^{m}$ is the diagonal action (i.e. $\gamma \cdot(x, u)=\left(\gamma \cdot x, \rho_{i}(\gamma)(u)\right)$, for $\gamma \in \Gamma_{i}, x \in \widetilde{U}_{i}$ and for $u \in \mathbb{R}^{m}$ ), and the map

$$
\Psi_{i}: \widetilde{V}_{i}=\widetilde{U}_{i} \times \mathbb{R}^{m} \longrightarrow E_{\mid U_{i}}:=\pi^{-1}\left(U_{i}\right)
$$

is such that $\pi_{\mid V_{i}} \circ \Psi_{i}=\varphi_{i} \circ \operatorname{pr}_{1}$, where $\operatorname{pr}_{1}: \widetilde{U}_{i} \times \mathbb{R}^{m} \longrightarrow \widetilde{U}_{i}$ is the natural projection, $\Psi_{i}$ is $\Gamma_{i}$-invariant for the action of $\Gamma_{i}$ on $\widetilde{U}_{i} \times \mathbb{R}^{m}$, and it induces a homeomorphism

$$
\Gamma_{i} \backslash\left(\widetilde{U}_{i} \times \mathbb{R}^{m}\right) \cong E_{\mid U_{i}}
$$

ii) If $\left(U_{i}, \widetilde{U}_{i}, \Gamma_{i}, \varphi_{i}\right)$ and $\left(U_{j}, \widetilde{U}_{j}, \Gamma_{j}, \varphi_{j}\right)$ are two orbifold charts on $X$, with $U_{i} \subset U_{j}$, and $\rho_{i j}: \widetilde{U}_{i} \longrightarrow \widetilde{U}_{j}$ is a change of charts, then there exists a differentiable map, called transition map

$$
g_{i j}: \widetilde{U}_{i} \longrightarrow \mathrm{GL}\left(\mathbb{R}^{m}\right) \text {, }
$$

and a change of charts $\lambda_{i j}: \widetilde{V}_{i}=\widetilde{U}_{i} \times \mathbb{R}^{m} \longrightarrow \widetilde{V}_{j}=\widetilde{U}_{j} \times \mathbb{R}^{m}$ on $E$, such that

$$
\lambda_{i j}(x, u)=\left(\rho_{i j}(x), g_{i j}(x)(u)\right),
$$

for all $(x, u) \in \widetilde{U}_{i} \times \mathbb{R}^{m}$.

Note that if $\pi: E \longrightarrow X$ is an orbifold vector bundle, and $p \in X$, then the fiber $\pi^{-1}(p)$ is not always a vector space. In fact, if $\left(U_{i}, \widetilde{U}_{i}, \Gamma_{i}, \varphi_{i}\right)$ is an orbifold chart on $X$ around $p=\varphi_{i}(x) \in X$, then

$$
\pi^{-1}(p) \cong \Gamma_{p} \backslash\left(x \times \mathbb{R}^{m}\right) \cong \Gamma_{p} \backslash \mathbb{R}^{m}
$$

where $\Gamma_{p}=\Gamma_{i}(x)$ is the isotropy group of $p$. Thus, $\pi^{-1}(p) \cong \mathbb{R}^{m}$ if $p$ is a regular point $\left(\Gamma_{p}=\mathrm{Id}\right)$ of $X$, but $\pi^{-1}(p)$ is not a vector space when $p$ is a singular point.

Definition 3.6. A section (or orbifold smooth section) of an orbifold vector bundle $\pi: E \longrightarrow X$ is an orbifold map $s: X \longrightarrow E$ such that $\pi \circ s=1_{X}$. Therefore, if $\left\{\left(U_{i}, \widetilde{U}_{i}, \Gamma_{i}, \varphi_{i}\right)\right\}$ is an atlas on $X$, then $s$ consists of a family of smooth maps $\left\{s_{i}: \widetilde{U}_{i} \longrightarrow\right.$ $\left.\mathbb{R}^{m}\right\}$, such that every $s_{i}$ is $\Gamma_{i}$-equivariant and compatible with the changes of charts on 
$X$ (in the sense of Definition [3.4). We denote the space of (orbifold smooth) sections of $E$ by $\mathcal{C}^{\infty}(E)$.

To construct the orbifold tangent bundle $T X$ of an orbifold $X$, of dimension $n$, we continue to use the notation of Definition 3.5. We define the orbifold charts and the transition maps for $T X$ as follows. For each orbifold chart $\left(U_{i}, \widetilde{U}_{i}, \Gamma_{i}, \varphi_{i}\right)$ of $X$, we consider the tangent bundle $T \widetilde{U}_{i}$ over $\widetilde{U}_{i}$, so $T \widetilde{U}_{i} \cong \widetilde{U}_{i} \times \mathbb{R}^{n}$. Take $\rho_{i}: \Gamma_{i} \longrightarrow \operatorname{GL}\left(\mathbb{R}^{n}\right)$ the homomorphism given by the action of $\Gamma_{i}$ on $\mathbb{R}^{n}$. Then $\left(E_{\mid U_{i}}, \widetilde{U}_{i} \times \mathbb{R}^{n}, \Gamma_{i}, \rho_{i}, \Psi_{i}\right)$ is an orbifold chart for $T X$, where $E_{\mid U_{i}}=\Gamma_{i} \backslash T \widetilde{U}_{i}$. Moreover, if $\rho_{i j}: \widetilde{U}_{i} \longrightarrow \widetilde{U}_{j}$ is a change of charts for $X$, the transition map

$$
g_{i j}: \widetilde{U}_{i} \longrightarrow \mathrm{GL}\left(\mathbb{R}^{n}\right)
$$

for $T X$ is such that $g_{i j}(x)$ is the Jacobian matrix of the map $\rho_{i j}$ at the point $x \in \widetilde{U}_{i}$. Therefore $T X$ is a $2 n$-dimensional orbifold, and the natural projection $\pi: T X \longrightarrow X$ defines a smooth map of orbifolds, with fibers $\pi^{-1}(p) \cong \Gamma_{p} \backslash\left(x \times \mathbb{R}^{n}\right) \cong \Gamma_{p} \backslash \mathbb{R}^{n}$, for $p \in X$. Therefore, one can consider tangent vectors to $X$ at the point $p \in X$ if $p$ is a regular point.

The orbifold cotangent bundle $T^{*} X$ and the orbifold tensor bundles are constructed similarly. Thus, one can consider Riemannian metrics, almost complex structures, orbifold forms, connections, etc.

An (orbifold) Riemannian metric $g$ on $X$ is a positive definite symmetric tensor in $T^{*} X \otimes T^{*} X$. This is equivalent to have, for each orbifold chart $\left(U_{i}, \widetilde{U}_{i}, \Gamma_{i}, \varphi_{i}\right)$ on $X$, a Riemannian metric $g_{i}$ on the open set $\widetilde{U}_{i}$ that is invariant under the action of $\Gamma_{i}$ on $\widetilde{U}_{i}\left(\Gamma_{i}\right.$ acts on $\widetilde{U}_{i}$ by isometries), and the change of charts $\rho_{i j}: \widetilde{U}_{i} \longrightarrow \widetilde{U}_{j}$ are isometries, that is $\rho_{i j}^{*}\left(\left.g_{j}\right|_{\rho_{i j}\left(\widetilde{U}_{i}\right)}\right)=g_{i}$.

An (orbifold) almost complex structure $J$ on $X$ is an endomorphism $J: T X \longrightarrow T X$ such that $J^{2}=-\mathrm{Id}$. Thus, $J$ is determined by an almost complex structure $J_{i}$ on $\widetilde{U}_{i}$, for every orbifold chart $\left(U_{i}, \widetilde{U}_{i}, \Gamma_{i}, \varphi_{i}\right)$ on $X$, such that the action of $\Gamma_{i}$ on $\widetilde{U}_{i}$ is by biholomorphic maps, and any change of charts $\rho_{i j}: \widetilde{U}_{i} \longrightarrow \widetilde{U}_{j}$ is a holomorphic embedding.

An orbifold $p$-form $\alpha$ on $X$ is a section of $\bigwedge^{p} T^{*} X$. This means that, for each orbifold chart $\left(U_{i}, \widetilde{U}_{i}, \Gamma_{i}, \varphi_{i}\right)$ on $X$, we have a differential $p$-form $\alpha_{i}$ on the open set $\widetilde{U}_{i}$, such that every $\alpha_{i}$ is $\Gamma_{i}$-invariant (i.e. $\gamma_{i}^{*}\left(\alpha_{i}\right)=\alpha_{i}$, for $\gamma_{i} \in \Gamma_{i}$ ), and any change of charts $\rho_{i j}: \widetilde{U}_{i} \longrightarrow \widetilde{U}_{j}$ satisfies $\rho_{i j}^{*}\left(\alpha_{j}\right)=\alpha_{i}$.

The space of $p$-forms on $X$ is denoted by $\Omega_{\text {orb }}^{p}(X)$. The wedge product of orbifold forms and the exterior differential $d$ on $X$ are well defined. Thus, we have

$$
d: \Omega_{\text {orb }}^{p}(X) \longrightarrow \Omega_{\text {orb }}^{p+1}(X) .
$$

The constant sheaf $\mathbb{R}$ has a resolution

$$
0 \longrightarrow \mathbb{R} \longrightarrow \Omega_{\text {orb }}^{0} \longrightarrow \Omega_{\text {orb }}^{1} \longrightarrow \ldots
$$

where $\Omega_{\text {orb }}^{p}$ is the sheaf of smooth sections of $\bigwedge^{p} T^{*} X$. To prove that this is a resolution, it is enough to prove that it is exact over any neighborhood of the form $U=\widetilde{U} / \Gamma$. As the 
group $\Gamma$ is finite, it is conjugate to a subgroup of $\varnothing(n)$, so we can assume that $\Gamma \subset \varnothing(n)$. We take $\widetilde{U}=B_{\epsilon}(0)$ (the ball in $\mathbb{R}^{n}$ of radius $\epsilon$ around the origin). Then

$$
0 \longrightarrow \mathbb{R} \longrightarrow \Omega^{0}(\widetilde{U}) \longrightarrow \Omega^{1}(\widetilde{U}) \longrightarrow \ldots
$$

is exact, and taking the $\Gamma$-invariant forms, the sequence in (3.2) is exact as well. (The functor $V \mapsto V^{\Gamma}$ that sends any vector space $V$ with a $\Gamma$-action, to its $\Gamma$-invariant part, is an exact functor.) Since (3.2) is exact, the cohomology of the complex $\left(\Omega_{\text {orb }}^{*}(X), d\right)$ is isomorphic to the singular cohomology $H^{*}(X, \mathbb{R})$.

We can see more explicitly this isomorphism with duality by pairing with homology classes in singular homology $H_{*}(X, \mathbb{R})$. Recall that we have a CW-complex structure for $X$ such that the singular sets $S_{H}=\left\{p \in X \mid \Gamma_{p}=H\right\}$ are CW-subcomplexes. Then for an orbifold $k$-form $\alpha$ on $X$ and a $k$-cell $D \subset X$, we have an integration map $\int_{D} \alpha$. This is defined as follows: we can assume that $D$ is inside an orbifold chart $(U, \widetilde{U}, \Gamma, \varphi)$. Let $D \subset \bar{S}_{H}$, where $H$ is some isotropy group, and assume that the interior of $D$ lies in $S_{H}$. Under the quotient map $\pi: \widetilde{U} \rightarrow U$, the preimage of $\pi^{-1}\left(S_{H} \cap U\right)$ is contained in a linear subspace, and the map $\pi: \pi^{-1}\left(S_{H} \cap U\right) \rightarrow S_{H} \cap U$ is $|H|: 1$. We define $\int_{D} \alpha=\frac{|H|}{|\Gamma|} \int_{\pi^{-1}(D)} \widetilde{\alpha}$, where $\widetilde{\alpha} \in \Omega^{k}(\widetilde{U})$ is the representative of $\alpha$ in the orbifold chart. It is easily seen that this is compatible with the orbifold changes of charts, and that it satisfies an orbifold version of Stokes theorem.

Remark 3.7. Suppose that $X=M / \Gamma$ is an oriented effective global orbifold, that is $X$ is the quotient of a smooth manifold $M$ by a finite group $\Gamma$ acting smoothly and effectively on $M$. Then, the definition of orbifold forms implies that any $\Gamma$-invariant differential $k$-form $\alpha$ on $M$ defines an orbifold $k$-form $\widehat{\alpha}$ on $X$, and vice-versa. Moreover, it is straightforward to check that the exterior derivative on $M$ preserves $\Gamma$-invariance. Thus, if $\left(\Omega^{k}(M)\right)^{\Gamma}$ denotes the space of the $\Gamma$-invariant differential $k$-form on $M$, and $H^{k}(M, \mathbb{R})^{\Gamma} \subset H^{k}(M, \mathbb{R})$ is the subspace of the cohomology classes of degree $k$ on $M$ such that each of these classes has a representative that is a $\Gamma$-invariant differential $k$-form, then we have

$$
\Omega_{\text {orb }}^{k}(X)=\left(\Omega^{k}(M)\right)^{\Gamma}, \quad H^{k}(X, \mathbb{R})=H^{k}(M, \mathbb{R})^{\Gamma} .
$$

For any compact supported orbifold $n$-form $\widehat{\alpha}$ on $X$, which is by definition a $\Gamma$-invariant compact supported differential $n$-form $\alpha$ on $M$, the integration of $\widehat{\alpha}$ on $X$ is defined by

$$
\int_{X} \widehat{\alpha}=|\Gamma| \int_{M} \alpha
$$

where $|\Gamma|$ is the order of the group $\Gamma$. More generally, one can extend the notion of integration to arbitrary orbifolds by working in orbifold charts via a partition of unity ([1, page 34], [35]).

Definition 3.8. Let $X$ be an orbifold. A minimal model for $X$ is a minimal model $(\bigwedge V, d)$ for the DGA $\left(\Omega_{\text {orb }}^{*}(X), d\right)$. The orbifold $X$ is formal if its minimal model is formal (see Section 2).

Proposition 3.9. Let $(\bigwedge V, d)$ be the minimal model of an orbifold $X$. Then $H^{*}(\bigwedge V)=$ $H^{*}(X, \mathbb{R})$, where the latter means singular cohomology with real coefficients. 
For a simply connected orbifold $X$, the dual of the real homotopy vector space $\pi_{i}(X) \otimes \mathbb{R}$ is isomorphic to the space $V^{i}$ of generators in degree $i$, for any $i$, where $\pi_{i}(X)$ is the homotopy group of order $i$ of the underlying topological space in $X$. In fact, the proof given in [11] for simply connected manifolds, also works for simply connected orbifolds (that is, orbifolds for which the topological space $X$ is simply connected).

Moreover, the proof of Theorem 2.3 given in [16] only uses that the cohomology $H^{*}(M)$ is a Poincaré duality algebra. By [35], we know that the singular cohomology of an orbifold also satisfies a Poincaré duality. Thus, Theorem 2.3 also holds for compact connected orientable orbifolds. Hence, we have the following lemma.

Lemma 3.10. Any simply connected compact orbifold of dimension at most 6 is formal.

The notion of formality is also defined for CW-complexes which have a minimal model $(\bigwedge V, d)$. Such a minimal model is constructed as the minimal model associated to the differential complex of piecewise-linear polynomial forms [14, 19]. In particular, we have a minimal model $(\bigwedge V, d)$ for orbifolds.

\section{ElLiptic DifFERENTIAL OPERATORS ON ORBIFOLDS}

Here we study elliptic differential operators on complex orbifolds by adapting to these spaces the elliptic operator theory on complex manifolds [43, Chapter IV].

A complex orbifold, of complex dimension $n$, is an orbifold $X$ whose orbifold charts are of the form $\left\{\left(U_{i}, \widetilde{U}_{i}, \Gamma_{i}, \varphi_{i}\right)\right\}$, where $\widetilde{U}_{i} \subset \mathbb{C}^{n}, \Gamma_{i} \subset \mathrm{GL}(n, \mathbb{C})$ is a finite group acting on $\widetilde{U}_{i}$ by biholomorphisms, and all the changes of charts $\rho_{i j}: \widetilde{U}_{i} \longrightarrow \widetilde{U}_{j}$ are holomorphic embeddings. Thus, any complex orbifold has associated an almost complex structure $J$.

If $X$ and $Y$ are complex orbifolds, a map $f: X \longrightarrow Y$ is said to be an orbifold holomorphic map (or holomorphic map) if $f$ is a continuous map between the underlying topological spaces, and for every point $p \in X$ there are orbifold charts $\left(U_{i}, \widetilde{U}_{i}, \Gamma_{i}, \varphi_{i}\right)$ and $\left(V_{i}, \widetilde{V}_{i}, \Upsilon_{i}, \psi_{i}\right)$ for $p$ and $f(p)$, respectively, with $f\left(U_{i}\right) \subset V_{i}$, and a holomorphic map $\widetilde{f}_{i}: \widetilde{U}_{i} \longrightarrow \widetilde{V}_{i}$ such that $\widetilde{f}_{i}$ is $\Gamma_{i}$-equivariant and compatible with changes of charts (in the sense of Definition 3.4).

Similarly to orbifold vector bundles, one can define complex orbifold vector bundles. Let $X$ be a complex orbifold, of complex dimension $n$. A complex orbifold vector bundle over $X$ and fiber $\mathbb{C}^{m}$ consists of a complex orbifold $E$, of complex dimension $m+n$, and a holomorphic orbifold map

$$
\pi: E \longrightarrow X
$$

such that the atlas on $E$ has charts of the type $\left(E_{\mid U_{i}}, \widetilde{U}_{i} \times \mathbb{C}^{m}, \Gamma_{i}, \rho_{i}, \Psi_{i}\right)$, where $\rho_{i}: \Gamma_{i} \longrightarrow$ $\mathrm{GL}\left(\mathbb{C}^{m}\right)$ is a homomorphism, and

$$
\Psi_{i}: \widetilde{U}_{i} \times \mathbb{C}^{m} \longrightarrow E_{\mid U_{i}}:=\pi^{-1}(U)
$$

is a $\Gamma_{i}$-invariant map, for the diagonal action of $\Gamma_{i}$ on $\widetilde{U}_{i} \times \mathbb{C}^{m}$ (the group $\Gamma_{i}$ acts on $\mathbb{C}^{m}$ via $\left.\rho_{i}\right)$, with $\Gamma_{i} \backslash\left(\widetilde{U}_{i} \times \mathbb{C}^{m}\right) \cong E_{\mid U_{i}}$.

A Hermitian metric $h$ on $X$ is a collection $\left\{h_{i}\right\}$, where each $h_{i}$ is a Hermitian metric on the open set $\widetilde{U}_{i}$ of the (complex) orbifold chart $\left(U_{i}, \widetilde{U}_{i}, \Gamma_{i}, \varphi_{i}\right)$ on $X$, such that every $h_{i}$ is $\Gamma_{i}$-invariant, and all the changes of charts $\rho_{i j}: \widetilde{U}_{i} \longrightarrow \widetilde{U}_{j}$ are given by holomorphic 
and isometric embeddings. A slight modification of the usual partition of unity argument shows that every complex orbifold has a Hermitian metric [27.

Complex orbifold forms on a complex orbifold and the orbifold Dolbeault cohomology will be considered in Section 5 .

Let $E \rightarrow X$ be a complex orbifold vector bundle endowed with a Hermitian metric. A Hermitian connection $\nabla$ on $E$ is defined to be a collection $\left\{\nabla_{i}\right\}$, where each $\nabla_{i}$ is a $\Gamma_{i^{-}}$ equivariant Hermitian connection on $\widetilde{U}_{i}$, for every complex orbifold chart $\left(U_{i}, \widetilde{U}_{i}, \Gamma_{i}, \varphi_{i}\right)$ on $X$, and such that $\nabla_{i}$ is compatible with changes of charts. Using $\nabla$, we can define Sobolev norms on sections of $E$. For a section $s$ supported on a chart $U_{i}$, define

$$
\|s\|_{W^{m}\left(\left.E\right|_{U_{i}}\right)}:=\frac{1}{\left|\Gamma_{i}\right|}\|s\|_{W^{m}\left(\widetilde{U}_{i}\right)}
$$

where $W^{m}$ denotes the usual Sobolev $m$-norm. That is, $\|s\|_{W^{m}\left(\widetilde{U}_{i}\right)}=\sum_{k=0}^{m}\left\|\nabla_{i}^{k} s\right\|_{L^{2}}$. For orbifold sections $s$ of $E$, we define $\|s\|_{W^{m}(E)}=\sum_{i}\left\|\rho_{i} s\right\|_{W^{m}\left(\left.E\right|_{U_{i}}\right)}$, where $\left\{U_{i}\right\}$ is a covering of $X$ by orbifold charts, and $\left\{\rho_{i}\right\}$ a subordinated (orbifold) partition of unity. The space $W^{m}(E)$ is the completion with respect to the $W^{m}$-norm of the space of (orbifold smooth) sections. In particular, $W^{0}(E)=L^{2}(E)$. The Sobolev embedding theorem and Rellich's lemma hold for orbifolds (the proof in [43, Chapter IV.1] can be extended to orbifolds verbatim).

A differential operator $L \in \operatorname{Diff}_{k}(E, F)$ of order $k$ between complex vector bundles $E$ and $F$ is a linear operator which is on an orbifold chart $\left(U_{i}, \widetilde{U}_{i}, \Gamma_{i}, \varphi_{i}\right)$ of the form

$$
L=\sum_{|\sigma| \leq k} a_{\sigma}(x) \frac{D^{|\sigma|}}{D^{\sigma} x},
$$

where $a_{\sigma}(x) \in \operatorname{Hom}(E, F)$ is defined on each $\widetilde{U}_{i}$ and it is $\Gamma_{i}$-equivariant. The symbol of $L$ is defined as

$$
\sigma_{k}(L)(x, \xi)=\sum_{|\sigma|=k} a_{\sigma}(x) \xi^{\sigma}
$$

for $x \in \widetilde{U}_{i}, \xi \in \mathbb{R}^{n}$. It is easily seen that this defines a symbol $\sigma_{k}(L)(x, \xi)$, for $x \in \widetilde{U}_{i}$ and $\xi \in T_{x}^{*} \widetilde{U}_{i}$, which is $\Gamma_{i}$-equivariant, that is, an orbifold section of the orbifold bundle $\operatorname{Hom}(E, F) \otimes\left(T^{*} X\right)^{\otimes k}$. We say that $L$ is an elliptic operator if the symbol of $L$ is an isomorphism for any $\xi \neq 0$.

The adjoint $L^{*}$ of a differential operator $L \in \operatorname{Diff}_{k}(E, F)$ is the operator defined by:

$$
\langle L(s), t\rangle=\left\langle s, L^{*}(t)\right\rangle,
$$

for any orbifold sections $s, t$ of $E, F$, respectively. It turns out that $L^{*} \in \operatorname{Diff}_{k}(F, E)$. For checking this, we go to an orbifold chart $\left(U_{i}, \widetilde{U}_{i}, \Gamma_{i}, \varphi_{i}\right)$. Then $L$ is written as (4.1). Then the equality (4.2), for compactly supported $\Gamma_{i}$-equivariant sections on $\widetilde{U}_{i}$, shows that $L^{*}$ has the form (4.1) for suitable coefficients $a_{\sigma}(x) \in \operatorname{Hom}(F, E)$, also $\Gamma_{i}$-equivariant. An operator $L \in \operatorname{Diff}_{k}(E):=\operatorname{Diff}_{k}(E, E)$ is called self-adjoint if $L^{*}=L$.

Theorem 4.1. Let $L \in \operatorname{Diff}_{k}(E)$ be self-adjoint and elliptic. Let

$$
\mathcal{H}_{L}(E)=\left\{v \in \mathcal{C}^{\infty}(E) \mid L(v)=0\right\} .
$$

Then there exist linear mappings $H_{L}, G_{L}: \mathcal{C}^{\infty}(E) \longrightarrow \mathcal{C}^{\infty}(E)$ such that 
(1) $H_{L}\left(\mathcal{C}^{\infty}(E)\right)=\mathcal{H}_{L}$ and $\operatorname{dim} \mathcal{H}_{L}(E)<\infty$,

(2) $L \circ G_{L}+H_{L}=G_{L} \circ L+H_{L}=$ Id,

(3) $H_{L}, G_{L}$ extend to bounded operators in $L^{2}(E)$, and

(4) $\mathcal{C}^{\infty}(E)=\mathcal{H}_{L}(E) \oplus G_{L} \circ L\left(\mathcal{C}^{\infty}(E)\right)=\mathcal{H}_{L}(E) \oplus L \circ G_{L}\left(\mathcal{C}^{\infty}(E)\right)$, with the decomposition being orthogonal with respect to the $L^{2}$-metric.

Proof. The theory in Chapter VI.3 of [43] works for orbifolds. A pseudo-differential operator is a linear operator $L$ which is locally of the form

$$
u(x) \longmapsto L(p) u(x)=\int p(x, \xi) \widehat{u}(\xi) e^{i\langle x, \xi\rangle} d \xi
$$

for compactly supported $u(x)$, where $p(x, \xi)$ is a $\Gamma$-invariant function on $T^{*} \widetilde{U}=\widetilde{U} \times \mathbb{R}^{n}$ such that the growth conditions in Definition 3.1 of [43, Chapter VI] hold. Note that $L(p)$ takes $\Gamma$-equivariant sections to $\Gamma$-equivariant sections. If we decompose $\mathcal{C}^{\infty}(\widetilde{U})=$ $\mathcal{C}^{\infty}(\widetilde{U})^{\Gamma} \oplus D$, where $D=\left\{s \mid \sum_{g \in \Gamma} g^{*} s=0\right\}$, then $L(p)$ maps $D$ to $D$.

A pseudo-differential operator

$$
L: \mathcal{C}^{\infty}(E) \longrightarrow \mathcal{C}^{\infty}(E)
$$

is of order $k$ if it extends continuously to $L: W^{m}(E) \longrightarrow W^{m+k}(E)$ for every $m$. Note that locally, $L$ maps $\Gamma$-equivariant sections of $W^{m}(\widetilde{U})$ to $\Gamma$-equivariant sections of $W^{m+k}(\widetilde{U})$. In particular, a differential operator of order $m$ is a pseudo-differential operator of order $m$.

First, using the ellipticity of $L$, one constructs a pseudo-differential operator $\widetilde{L}$, such that $L \circ \widetilde{L}-$ Id and $\widetilde{L} \circ L-$ Id are of order -1 . With this, one can check the regularity of the solutions of the equation $L v=0$, that is

$$
\mathcal{H}_{L}(E)_{m}=\left\{v \in W^{m}(E) \mid L v=0\right\} \subset \mathcal{C}^{\infty}(E),
$$

so that $\mathcal{H}_{L}(E)=\mathcal{H}_{L}(E)_{m}$ for all $m$. Using Rellich's lemma, this proves that $\mathcal{H}_{L}(E)$ is of finite dimension. Now $H_{L}$ is defined as projection onto $\mathcal{H}_{L}(E)$, and $G_{L}$ is defined as the inverse of $L$ on the orthogonal complement to $\mathcal{H}_{L}(E)$ and zero on $\mathcal{H}_{L}(E)$. With this, it turns out that $G_{L}$ is an operator of negative order. The rest of the assertions are now straightforward.

Let $E_{0}, E_{1}, \ldots, E_{N}$ be a collection of complex orbifold vector bundles over $X$. A sequence of differential operators

$$
\mathcal{C}^{\infty}\left(E_{0}\right) \stackrel{L_{0}}{\longrightarrow} \mathcal{C}^{\infty}\left(E_{1}\right) \stackrel{L_{1}}{\longrightarrow} \mathcal{C}^{\infty}\left(E_{2}\right) \stackrel{L_{2}}{\longrightarrow} \ldots \stackrel{L_{N-1}}{\longrightarrow} \mathcal{C}^{\infty}\left(E_{N}\right)
$$

is an elliptic complex if $L_{i} \circ L_{i-1}=0, i=1, \cdots, N-1$, and the sequence of symbols

$$
0 \longrightarrow\left(E_{0}\right)_{x} \stackrel{\sigma\left(L_{0}\right)}{\longrightarrow}\left(E_{1}\right)_{x} \stackrel{\sigma\left(L_{1}\right)}{\longrightarrow}\left(E_{2}\right)_{x} \stackrel{\sigma\left(L_{2}\right)}{\longrightarrow} \ldots \stackrel{\sigma\left(L_{N-1}\right)}{\longrightarrow}\left(E_{N}\right)_{x} \longrightarrow 0
$$

is exact for all $x \in X, \xi \neq 0$. The cohomology groups of the complex are defined to be

$$
H^{q}(E):=\frac{\operatorname{ker} L_{q}}{\operatorname{im} L_{q-1}} .
$$

Writing $E=\bigoplus_{i=1}^{N} E_{i}, L=\sum_{i=1}^{N-1} L_{i}$, and

$$
\Delta=L^{*} L+L L^{*}
$$


with respect to some fixed Hermitian metric on every $E_{i}, 0 \leq i \leq N$, we have an elliptic operator $\Delta: \mathcal{C}^{\infty}(E) \longrightarrow \mathcal{C}^{\infty}(E)$. Note that $\Delta: \mathcal{C}^{\infty}\left(E_{i}\right) \longrightarrow \mathcal{C}^{\infty}\left(E_{i}\right)$, for all $i=0,1, \ldots, N$. We denote

$$
\mathcal{H}^{j}(E)=\operatorname{ker}\left(\left.\Delta\right|_{E_{j}}\right) .
$$

The following is an analogue of Theorem 5.2 in [43, Chapter V].

Theorem 4.2. Let $\left(\mathcal{C}^{\infty}(E), L\right)$ be an elliptic complex equipped with an inner product. Then the following statements hold:

(1) There is an orthogonal decomposition

$$
\mathcal{C}^{\infty}(E)=\mathcal{H}(E) \oplus L L^{*} G\left(\mathcal{C}^{\infty}(E)\right) \oplus L^{*} L G\left(\mathcal{C}^{\infty}(E)\right) .
$$

(2) $\mathrm{Id}=H+\Delta G=H+G \Delta, H G=G H=H \Delta=\Delta H=0, L \Delta=\Delta L, L^{*} \Delta=\Delta L^{*}$, $L G=G L, L^{*} G=G L^{*}, L H=H L=L^{*} H=H L^{*}=0$.

(3) $\operatorname{dim} \mathcal{H}^{j}(E)<\infty$, and there is a canonical isomorphism $\mathcal{H}^{j}(E) \cong H^{j}(E)$.

(4) $\Delta v=0 \Longleftrightarrow L v=L^{*} v=0$ for all $v \in \mathcal{C}^{\infty}(E)$.

The complex

$$
\Omega_{\text {orb }}^{0}(X) \stackrel{d}{\longrightarrow} \Omega_{\text {orb }}^{1}(X) \stackrel{d}{\longrightarrow} \Omega_{\text {orb }}^{2}(X) \stackrel{d}{\longrightarrow} \cdots \stackrel{d}{\longrightarrow} \Omega_{\text {orb }}^{n}(X)
$$

is elliptic. Hence Theorem 4.2 implies that

$$
H^{k}(X) \cong \mathcal{H}^{k}(X)=\operatorname{ker}\left(\Delta: \Omega_{o r b}^{k}(X) \longrightarrow \Omega_{o r b}^{k}(X)\right),
$$

where $\Delta=d d^{*}+d^{*} d$.

\section{KÄHLER ORBIFOLDS}

Let $X$ be a complex orbifold, of complex dimension $n$, with an atlas $\left\{\left(U_{i}, \widetilde{U}_{i}, \Gamma_{i}, \varphi_{i}\right)\right\}$. As for complex manifolds, we can consider orbifold complex forms on $X$. An orbifold complex $k$-form $\alpha$ on $X$ is given by a complex $k$-form $\alpha_{i}$ on the open set $\widetilde{U}_{i}$, for each orbifold chart $\left(U_{i}, \widetilde{U}_{i}, \Gamma_{i}, \varphi_{i}\right)$, and such that every $\alpha_{i}$ is $\Gamma_{i}$-invariant and preserved by all the change of charts. We say that $\alpha$ is bigraded of type $(p, q)$, with $k=p+q$, if each $\alpha_{i}$ is a $(p, q)$-form on $\widetilde{U}_{i}$. Denote by $\Omega_{o r b}^{p, q}(X)$ the space of orbifold $(p, q)$-forms on $X$. Then, we have the type decomposition of the exterior derivative $d=\partial+\bar{\partial}$, where

$$
\partial: \Omega_{o r b}^{p, q}(X) \longrightarrow \Omega_{\text {orb }}^{p+1, q}(X) \text { and } \bar{\partial}: \Omega_{o r b}^{p, q}(X) \longrightarrow \Omega_{o r b}^{p, q+1}(X) .
$$

The (orbifold) Dolbeault cohomology of $X$ is defined to be

$$
H^{p, q}(X):=\frac{\operatorname{ker}\left(\bar{\partial}: \Omega_{o r b}^{p, q}(X) \longrightarrow \Omega_{o r b}^{p, q+1}(X)\right)}{\bar{\partial}\left(\Omega_{o r b}^{p, q-1}(X)\right)} .
$$

Fix an orbifold Hermitian metric on $X$. For any $p \geq 0$, the complex

$$
0 \longrightarrow \Omega_{\text {orb }}^{p, 0}(X) \stackrel{\bar{\partial}}{\longrightarrow} \Omega_{\text {orb }}^{p, 1}(X) \stackrel{\bar{\partial}}{\longrightarrow} \Omega_{\text {orb }}^{p, 2}(X) \stackrel{\bar{\partial}}{\longrightarrow} \ldots \stackrel{\bar{\partial}}{\longrightarrow} \Omega_{\text {orb }}^{p, n}(X) \longrightarrow 0
$$

is elliptic, where $n$ is the complex dimension of $X$. Hence Theorem 4.2 implies that

$$
H^{p, q}(X) \cong \mathcal{H}^{p, q}(X)=\operatorname{ker}\left(\Delta_{\bar{\partial}}: \Omega_{\text {orb }}^{p, q}(X) \longrightarrow \Omega_{\text {orb }}^{p, q}(X)\right),
$$

where $\Delta_{\bar{\partial}}=\bar{\partial}_{\bar{\partial}}^{*}+\bar{\partial}^{*} \bar{\partial}$. 
Let $(X, J, h)$ be a complex Hermitian orbifold, with orbifold complex structure $J$ and Hermitian metric $h$. Thus, we have an orbifold Riemannian metric $g=\operatorname{Re} h$ and an orbifold 2 -form $\omega \in \Omega_{\text {orb }}^{1,1}(X)$ defined by

$$
\omega=\operatorname{Im} h
$$

Then, $\omega^{n} \neq 0$, where $n$ is the complex dimension of $X$.

Definition 5.1. A complex Hermitian orbifold $(X, h)$ is called Kähler orbifold if the associated fundamental form $\omega$ is closed, that is $d \omega=0$.

Proposition 5.2. For a compact Kähler orbifold,

$$
\Delta=2 \Delta \bar{\partial} .
$$

Therefore $\mathcal{H}^{k}(X)=\bigoplus_{p+q=k} \mathcal{H}^{p, q}(X)$.

Proof. This is true on the dense open subset of non-singular points of $X$ by Theorem 4.7 of [43, Chapter V]. So it holds everywhere on $X$.

Corollary 5.3. For a compact Kähler orbifold, $b_{k}(X)$ is even for $k$ odd.

Proof. Clearly, conjugation gives a map $\Omega_{\text {orb }}^{p, q}(X) \longrightarrow \Omega_{\text {orb }}^{q, p}(X)$ that commutes with $\Delta$ (as this is a real operator). Therefore, the induced map $\mathcal{H}^{p, q}(X) \longrightarrow \mathcal{H}^{q, p}(X)$ is an isomorphism. In particular, $h^{p, q}(X)=h^{q, p}(X)$, where $h^{p, q}(X)=\operatorname{dim} H^{p, q}(X)$. Thus, $b_{k}(X)=\sum_{k=p+q} h^{p, q}(X)$ is even for $k$ odd.

\section{Lemma 5.4.}

(1) Take $\alpha \in \Omega_{\text {orb }}^{p, q}(X)$ with $\partial \alpha=0$. If $\alpha=\bar{\partial} \beta$ for some $\beta$, then there exists $\psi$ such that $\alpha=\partial \bar{\partial} \psi$.

(2) Take $\alpha \in \Omega_{\text {orb }}^{p, q}(X)$ with $\bar{\partial} \alpha=0$. If $\alpha=\partial \beta$ for some $\beta$, then there exists $\psi$ such that $\alpha=\partial \bar{\partial} \psi$.

Proof. Using Theorem 4.2,

$$
\alpha=H \alpha+\Delta_{\bar{\partial}} G \alpha=H \alpha+\bar{\partial} \bar{\partial}^{*} G \alpha+\bar{\partial}^{*} \bar{\partial} G \alpha,
$$

where $G=G_{\bar{\partial}}$ is the Green's operator associated to $\bar{\partial}$. As $\alpha=\bar{\partial} \beta$, the cohomology class represented by $\alpha$ vanishes, so $H \alpha=0$. Then, since $G$ commutes with $\bar{\partial}$, we have $\bar{\partial} G \alpha=G \bar{\partial} \alpha=0$. Hence $\alpha=\bar{\partial} \bar{\partial}^{*} G \alpha=\bar{\partial} G\left(\bar{\partial}^{*} \alpha\right)$.

Now $\bar{\partial}^{*}=\sqrt{-1}[\Lambda, \partial]$, where $\Lambda=L_{\omega}^{*}$ and $L_{\omega}(\beta)=\omega \wedge \beta$. So $\bar{\partial}^{*} \alpha=-\sqrt{-1} \partial \Lambda \alpha$, because $\partial \alpha=0$. Hence $\alpha=\bar{\partial} G(-\sqrt{-1} \partial \Lambda \alpha)=-\sqrt{-1} \bar{\partial} \partial(G \Lambda \alpha)$. Therefore, taking $\psi=-\sqrt{-1} G \Lambda \alpha$, we conclude the proof of the first part.

The proof of the second part is identical.

Theorem 5.5. Let $X$ be a compact Kähler orbifold. Then $X$ is formal.

Proof. We have to show that $\left(\Omega_{o r b}^{*}(X), d\right)$ and $\left(H^{*}(X), 0\right)$ are quasi-isomorphic differential graded commutative algebras (DGA).

Consider the DGA (ker $\partial, \bar{\partial})$. We will show that

$$
\imath:(\operatorname{ker} \partial, \bar{\partial}) \hookrightarrow\left(\Omega_{\text {orb }}^{*}(X), d\right)
$$


is a quasi-isomorphism. To prove surjectivity, we can take a $(p, q)$-form $\alpha$ which is $d$ closed (see Proposition 5.2). If $d \alpha=0$, then $\partial \alpha=0$ and $\bar{\partial} \alpha=0$. So $\alpha \in \operatorname{ker} \partial$ and $\imath^{*}[\alpha]=[\alpha]$. For injectivity, take $\alpha \in \operatorname{ker} \partial$ such that $\imath^{*}[\alpha]=0$. Then $\bar{\partial} \alpha=0$ and $\alpha=d \beta$, for some form $\beta$. Therefore, $\alpha=\partial \beta+\bar{\partial} \beta$. Thus we have $\bar{\partial}(\partial \beta)=0$. By Lemma 5.4, we have that $\partial \beta=\partial \bar{\partial} \psi$ for some $\psi$. Hence $\alpha=\bar{\partial} \beta+\partial \bar{\partial} \psi=\bar{\partial}(\beta-\partial \psi-\bar{\partial} \psi)$. Note that $\partial(\beta-\partial \psi-\bar{\partial} \psi)=\partial \beta-\partial \bar{\partial} \psi=0$, so $\beta-\partial \psi-\bar{\partial} \psi \in \operatorname{ker} \partial$.

Next we will show that the projection given by

$$
H:(\operatorname{ker} \partial, \bar{\partial}) \longrightarrow\left(\mathcal{H}_{\bar{\partial}}^{*}(X), 0\right)
$$

is a quasi-isomorphism.

Let $\alpha \in \operatorname{ker} \partial \cap \operatorname{ker} \bar{\partial}$. Then $\bar{\partial}^{*} \alpha=\sqrt{-1}[\Lambda, \partial] \alpha=-\sqrt{-1} \partial(\Lambda \alpha)$. So

$$
\alpha=H \alpha+G\left(\bar{\partial} \bar{\partial}^{*} \alpha+\bar{\partial}^{*} \bar{\partial} \alpha\right)=H \alpha-\sqrt{-1} G \bar{\partial} \partial(\Lambda \alpha)
$$

that is $\alpha=H \alpha+\partial \bar{\partial} \psi$, for some $\psi$. Therefore, if $H \alpha=0$, then $\alpha=\bar{\partial}(\partial \psi)$, with $\partial \psi \in \operatorname{ker} \partial$. This proves injectivity.

Now suppose $\alpha=H \alpha+\partial \bar{\partial} \psi$ and $\beta=H \beta+\partial \bar{\partial} \phi$. So

$$
\alpha \wedge \beta=H \alpha \wedge H \beta+\partial \bar{\partial} \Phi
$$

for some $\Phi$, hence $H(\alpha \wedge \beta)=H \alpha \wedge H \beta$. This implies that $H$ is a DGA map.

Finally, let us show surjectivity of $H$. Take $\alpha$ to be harmonic. Then $\bar{\partial} \alpha=0$ and $\bar{\partial}^{*} \alpha=0$. Since $\Delta=2 \Delta_{\bar{\partial}}$, we also have $d \alpha=0$ and $\partial \alpha=0$. So $H([\alpha])=\alpha$.

The hard Lefschetz property is proved in [42, but we shall give a proof with the current techniques for completeness.

Theorem 5.6. Let $(X, \omega)$ be a compact Kähler orbifold. Then the map

$$
L_{\omega}^{n-k}: H^{k}(X) \longrightarrow H^{2 n-k}(X)
$$

is an isomorphism for $0 \leq k \leq n$.

Proof. It is enough to see that (5.1) is onto, since by Poincaré duality both spaces have the same dimension. As $\left[L_{\omega}, \Delta\right]=0$, then $L_{\omega}$ sends harmonic forms to harmonic forms. Therefore we have to see that

$$
L_{\omega}^{n-k}: \mathcal{H}^{k}(X) \longrightarrow \mathcal{H}^{2 n-k}(X)
$$

is surjective. We shall prove this by induction on $k=0,1, \ldots, n$. Take a harmonic $(2 n-k)$-form $a$. By induction on $k$ applied to $L_{\omega}(a)$, we have that $L_{\omega}(a)=L_{\omega}^{n-k+2}(c)$ for a $(k-2)$-form $c$. Therefore $a^{\prime}=a-L_{\omega}^{n-k+1}(c)$ is primitive, $L_{\omega}\left(a^{\prime}\right)=0$. Let us see that the Lefschetz map is surjective for a primitive $a^{\prime}$.

We have that $\left[\Lambda, L_{\omega}\right]=n-p$ on $p$-forms. As $\Lambda a=0$, we have $L_{\omega}\left(\Lambda a^{\prime}\right)=(k-n) a^{\prime}$, so $\left(L_{\omega}\left(\Lambda a^{\prime}\right)\right)^{n-k}=c a^{\prime}$, for a constant $c$. Using repeatedly that $L_{\omega} \Lambda=\Lambda L_{\omega}+c$ Id, for (different constants $c^{\prime}$ s), we get that $L_{\omega}^{n-k} \Lambda^{n-k} a^{\prime}=c a^{\prime}$, for another constant $c$. The map

$$
L_{\omega}^{n-k}: \Omega_{\text {orb }}^{k}(X) \longrightarrow \Omega_{\text {orb }}^{2 n-k}(X)
$$

is an isomorphism (it is a bundle isomorphism). So the above constant $c$ is nonzero. Therefore, $a^{\prime}=L_{\omega}^{n-k}(b)$ with $b=\frac{1}{c} \Lambda^{n-k}\left(a^{\prime}\right)$ a harmonic $k$-form (since $\Lambda$ also sends harmonic forms to harmonic forms). This finishes the proof of the theorem. 


\section{Symplectic ORBifolds With No Kähler ORBIFOld STRUCTURE}

We shall include two examples of symplectic orbifolds, of dimensions 6 and 8, taken from the constructions in [5] and [17], which cannot admit the structure of an orbifold Kähler manifold. The first one because it does not satisfy the hard Lefschetz property, and the second one because it is non-formal. Both admit complex and symplectic (orbifold) structures.

Before going to those examples, let us recall the definition of a symplectic orbifold.

Definition 6.1. A symplectic orbifold $(X, \omega)$ consists of a $2 n$-dimensional orbifold $X$ and an orbifold 2-form $\omega$ such that $d \omega=0$ and $\omega^{n}>0$ everywhere.

Note that if $(M, \Omega)$ is a symplectic manifold, with symplectic form $\Omega$, and $\Gamma$ is a finite group acting effectively on $M$ and preserving $\Omega$, then $X=M / \Gamma$ is a symplectic orbifold. In fact, by Remark [3.7, $X=M / \Gamma$ is an orbifold, and the symplectic form $\Omega$ descends to $X$ via the natural projection $\pi: M \rightarrow X$. The map $\pi$ is differentiable in the orbifold sense (actually it is a submersion).

6.1. 6-dimensional example. Consider the complex Heisenberg group $H_{\mathbb{C}}$, that is the complex nilpotent Lie group of (complex) dimension 3 consisting of matrices of the form

$$
\left(\begin{array}{ccc}
1 & u_{2} & u_{3} \\
0 & 1 & u_{1} \\
0 & 0 & 1
\end{array}\right) .
$$

In terms of the natural (complex) coordinate functions $\left(u_{1}, u_{2}, u_{3}\right)$ on $H_{\mathbb{C}}$, we have that the complex 1-forms $\mu=d u_{1}, \nu=d u_{2}$ and $\theta=d u_{3}-u_{2} d u_{1}$ are left invariant, and

$$
d \mu=d \nu=0, \quad d \theta=\mu \wedge \nu .
$$

Let $\Lambda \subset \mathbb{C}$ be the lattice generated by 1 and $\zeta=e^{2 \pi i / 6}$, and consider the discrete subgroup $\Gamma \subset H_{\mathbb{C}}$ formed by the matrices in which $u_{1}, u_{2}, u_{3} \in \Lambda$. We define the compact (parallelizable) nilmanifold

$$
M=\Gamma \backslash H_{\mathbb{C}} .
$$

We can describe $M$ as a principal torus bundle

$$
T^{2}=\mathbb{C} / \Lambda \longrightarrow M \longrightarrow T^{4}=(\mathbb{C} / \Lambda)^{2}
$$

by the projection $\left(u_{1}, u_{2}, u_{3}\right) \longmapsto\left(u_{1}, u_{2}\right)$.

Consider the action of the finite group $\mathbb{Z}_{6}$ on $H_{\mathbb{C}}$ given by the generator

$$
\begin{aligned}
\rho: H_{\mathbb{C}} & \longrightarrow H_{\mathbb{C}} \\
\left(u_{1}, u_{2}, u_{3}\right) & \longmapsto\left(\zeta^{4} u_{1}, \zeta u_{2}, \zeta^{5} u_{3}\right) .
\end{aligned}
$$

For this action, clearly $\rho(p \cdot q)=\rho(p) \cdot \rho(q)$, for all $p, q \in H_{\mathbb{C}}$, where $\cdot$ denotes the natural group structure of $H_{\mathbb{C}}$. Moreover, we have $\rho(\Gamma)=\Gamma$. Thus, $\rho$ induces an action on the quotient $M=\Gamma \backslash H_{\mathbb{C}}$. Let $\rho: M \longrightarrow M$ be the $\mathbb{Z}_{6}$-action. The action on 1-forms is given by

$$
\rho^{*} \mu=\zeta^{4} \mu, \quad \rho^{*} \nu=\zeta \nu, \quad \rho^{*} \theta=\zeta^{5} \theta .
$$

Proposition 6.2. $X=M / \mathbb{Z}_{6}$ is a simply connected, compact, formal 6-orbifold admitting complex and symplectic structures. 
Proof. Since the $\mathbb{Z}_{6}$-action on $M$ is effective, the quotient space $X=M / \mathbb{Z}_{6}$ is an orbifold. (The singular points of $X$ are determined in [5, Section 4].) Clearly $X$ is compact since $M$ is compact. In [5, Proposition 6.1], it is proved that the 6-orbifold $X$ (denoted by $\widehat{M}$ in [5]) is simply connected. Then, $X$ is formal because any simply connected compact orbifold of dimension 6 is formal by Lemma 3.10.

The orbifold $X$ has a complex orbifold structure, as in Proposition 6.4. We define the complex 2-form $\omega$ on $M$ by

$$
\omega=-\sqrt{-1} \mu \wedge \bar{\mu}+\nu \wedge \theta+\bar{\nu} \wedge \bar{\theta} .
$$

Clearly, $\omega$ is a real closed 2 -form on $M$ such that $\omega^{3}>0$, so, $\omega$ is a symplectic form on $M$. Moreover, the form $\omega$ is $\mathbb{Z}_{6}$-invariant. Indeed, $\rho^{*} \omega=-i \mu \wedge \bar{\mu}+\zeta^{6} \nu \wedge \theta+\zeta^{-6} \bar{\nu} \wedge \bar{\theta}=\omega$. Therefore $X$ is a symplectic 6 -orbifold, with the symplectic form $\widehat{\omega}$ induced by $\omega$.

In order to prove that $X$ does not admit any Kähler structure, we are going to check that it does not satisfy the hard Lefschetz property for any symplectic form. We compute the cohomology of $X$. By a theorem of Nomizu theorem [32], the cohomology of the nilmanifold $M$ is:

$$
\begin{aligned}
H^{0}(M, \mathbb{C})= & \langle 1\rangle, \\
H^{1}(M, \mathbb{C})= & \langle[\mu],[\bar{\mu}],[\nu],[\bar{\nu}]\rangle, \\
H^{2}(M, \mathbb{C})= & \langle[\mu \wedge \bar{\mu}],[\mu \wedge \bar{\nu}],[\bar{\mu} \wedge \nu],[\nu \wedge \bar{\nu}],[\mu \wedge \theta],[\bar{\mu} \wedge \bar{\theta}],[\nu \wedge \theta],[\bar{\nu} \wedge \bar{\theta}]\rangle, \\
H^{3}(M, \mathbb{C})= & \langle[\mu \wedge \bar{\mu} \wedge \theta],[\mu \wedge \bar{\mu} \wedge \bar{\theta}],[\nu \wedge \bar{\nu} \wedge \theta],[\nu \wedge \bar{\nu} \wedge \bar{\theta}],[\mu \wedge \nu \wedge \theta],[\bar{\mu} \wedge \bar{\nu} \wedge \bar{\theta}] \\
& {[\mu \wedge \bar{\nu} \wedge \theta],[\mu \wedge \bar{\nu} \wedge \bar{\theta}],[\bar{\mu} \wedge \nu \wedge \theta],[\bar{\mu} \wedge \nu \wedge \bar{\theta}]\rangle, } \\
H^{4}(M, \mathbb{C})= & \langle[\mu \wedge \bar{\mu} \wedge \nu \wedge \theta],[\mu \wedge \bar{\mu} \wedge \bar{\nu} \wedge \bar{\theta}],[\bar{\mu} \wedge \nu \wedge \bar{\nu} \wedge \bar{\theta}],[\mu \wedge \nu \wedge \bar{\nu} \wedge \theta], \\
& {[\mu \wedge \bar{\mu} \wedge \theta \wedge \bar{\theta}],[\nu \wedge \bar{\nu} \wedge \theta \wedge \bar{\theta}],[\mu \wedge \bar{\nu} \wedge \theta \wedge \bar{\theta}],[\bar{\mu} \wedge \nu \wedge \theta \wedge \bar{\theta}]\rangle, } \\
H^{5}(M, \mathbb{C})= & \langle[\mu \wedge \bar{\mu} \wedge \nu \wedge \theta \wedge \bar{\theta}],[\mu \wedge \bar{\mu} \wedge \bar{\nu} \wedge \theta \wedge \bar{\theta}],[\mu \wedge \nu \wedge \bar{\nu} \wedge \theta \wedge \bar{\theta}],[\bar{\mu} \wedge \nu \wedge \bar{\nu} \wedge \theta \wedge \bar{\theta}]\rangle, \\
H^{6}(M, \mathbb{C})= & \langle[\mu \wedge \bar{\mu} \wedge \nu \wedge \bar{\nu} \wedge \theta \wedge \bar{\theta}]\rangle .
\end{aligned}
$$

According with (3.3), any $\mathbb{Z}_{6}$-invariant $k$-form on $M$ defines an orbifold $k$-form on $X$, and vice-versa. Moreover, the cohomology $H^{*}(X)=H^{*}(M)^{\mathbb{Z}_{6}}$ is:

$$
\begin{aligned}
& H^{0}(X, \mathbb{C})=\langle 1\rangle, \\
& H^{1}(X, \mathbb{C})=0, \\
& H^{2}(X, \mathbb{C})=\langle[\mu \wedge \bar{\mu}],[\nu \wedge \bar{\nu}],[\nu \wedge \theta],[\bar{\nu} \wedge \bar{\theta}]\rangle, \\
& H^{3}(X, \mathbb{C})=0 \\
& H^{4}(X, \mathbb{C})=\langle[\mu \wedge \bar{\mu} \wedge \nu \wedge \theta],[\mu \wedge \bar{\mu} \wedge \bar{\nu} \wedge \bar{\theta}],[\mu \wedge \bar{\mu} \wedge \theta \wedge \bar{\theta}],[\nu \wedge \bar{\nu} \wedge \theta \wedge \bar{\theta}]\rangle, \\
& H^{5}(X, \mathbb{C})=0 \\
& H^{6}(X, \mathbb{C})=\langle[\mu \wedge \bar{\mu} \wedge \nu \wedge \bar{\nu} \wedge \theta \wedge \bar{\theta}]\rangle,
\end{aligned}
$$

where we use the same notation for the $\mathbb{Z}_{6}$-invariant forms on $M$ and those induced on the orbifold $X$.

The cohomology class

$$
[\beta]=[\nu \wedge \bar{\nu}] \in H^{2}(X)
$$


satisfies the equation $[\beta] \wedge\left[\alpha_{1}\right] \wedge\left[\alpha_{2}\right]=0$ for any $\left[\alpha_{1}\right],\left[\alpha_{2}\right] \in H^{2}(X)$. Therefore this class is always in the kernel of

$$
L_{\omega^{\prime}}: H^{2}(X) \longrightarrow H^{4}(X),
$$

for any (orbifold) symplectic form $\omega^{\prime}$. So we have the following:

Proposition 6.3. The orbifold $X$ does not admit an orbifold Kähler structure since it does not satisfy the hard Lefschetz property for any symplectic form.

6.2. 8-dimensional example. Consider again the complex Heisenberg group $H_{\mathbb{C}}$ and set $G=H_{\mathbb{C}} \times \mathbb{C}$, where $\mathbb{C}$ is the additive group of complex numbers. We denote by $u_{4}$ the coordinate function corresponding to this extra factor. In terms of the natural (complex) coordinate functions $\left(u_{1}, u_{2}, u_{3}, u_{4}\right)$ on $G$, the complex 1-forms $\mu=d u_{1}, \nu=d u_{2}, \theta=$ $d u_{3}-u_{2} d u_{1}$ and $\eta=d u_{4}$ are left invariant, and

$$
d \mu=d \nu=d \eta=0, \quad d \theta=\mu \wedge \nu
$$

Let $\Lambda \subset \mathbb{C}$ be the lattice generated by 1 and $\zeta=e^{2 \pi \sqrt{-1} / 3}$, and consider the discrete subgroup $\Gamma \subset G$ formed by the matrices in which $u_{1}, u_{2}, u_{3}, u_{4} \in \Lambda$. We define the compact (parallelizable) nilmanifold

$$
M=\Gamma \backslash G .
$$

We can describe $M$ as a principal torus bundle

$$
T^{2}=\mathbb{C} / \Lambda \longrightarrow M \longrightarrow T^{6}=(\mathbb{C} / \Lambda)^{3},
$$

by the projection $\left(u_{1}, u_{2}, u_{3}, u_{4}\right) \longmapsto\left(u_{1}, u_{2}, u_{4}\right)$.

Now introduce the following action of the finite group $\mathbb{Z}_{3}$

$$
\begin{aligned}
\rho: G & \longrightarrow G \\
\left(u_{1}, u_{2}, u_{3}, u_{4}\right) & \longmapsto\left(\zeta u_{1}, \zeta u_{2}, \zeta^{2} u_{3}, \zeta u_{4}\right) .
\end{aligned}
$$

Note that $\rho(p \cdot q)=\rho(p) \cdot \rho(q)$, for $p, q \in G$, where the dot denotes the natural group structure of $G$. The map $\rho$ is a particular case of a homothetic transformation (by $\zeta$ in this case) which is well defined for all nilpotent simply connected Lie groups with graded Lie algebra. Moreover $\rho(\Gamma)=\Gamma$, therefore $\rho$ induces an action on the quotient $M=\Gamma \backslash G$. This action is free away from $3^{4}$ fixed points corresponding to $u_{i}=n /(1-\zeta)$, for $n=0,1$ and 2 .

The action on the forms is given by

$$
\rho^{*} \mu=\zeta \mu, \quad \rho^{*} \nu=\zeta \nu, \quad \rho^{*} \theta=\zeta^{2} \theta, \quad \rho^{*} \eta=\zeta \eta .
$$

Proposition 6.4. $X=M / \mathbb{Z}_{3}$ is an 8-orbifold admitting complex and symplectic structures.

Proof. Just as in Proposition 6.2, it turns out that $X$ is an 8 -orbifold since the $\mathbb{Z}_{3}$-action on $M$ is effective. The nilmanifold $M$ is a complex manifold whose complex structure $J$ coincides with the multiplication by $\sqrt{-1}$ on each tangent space $T_{p} M, p \in M$. Then one can check that $J$ commutes with the $\mathbb{Z}_{3}$-action $\rho$ on $M$, that is $\left(\rho_{*}\right)_{p} \circ J_{p}=J_{\rho(p)} \circ\left(\rho_{*}\right)_{p}$, for any point $p \in M$. Hence, $J$ induces a complex structure on the quotient $X=M / \mathbb{Z}_{3}$.

The complex 2-form

$$
\omega=\sqrt{-1} \mu \wedge \bar{\mu}+\nu \wedge \theta+\bar{\nu} \wedge \bar{\theta}+i \eta \wedge \bar{\eta}
$$


is actually a real form which is clearly closed and which has the property that $\omega^{4} \neq 0$. Thus $\omega$ is a symplectic form on $M$. Moreover, $\omega$ is $\mathbb{Z}_{3}$-invariant. Hence the space $X=$ $M / \mathbb{Z}_{3}$ is a symplectic orbifold, with the symplectic form $\widehat{\omega}$ induced by $\omega$.

The orbifold $X$ does not admit a Kähler orbifold structure because it is non-formal, as shown in the following theorem.

Theorem 6.5. The orbifold $X$ is non-formal.

Proof. We start by considering the nilmanifold $M$. Consider the following closed forms:

$$
\alpha=\mu \wedge \bar{\mu}, \quad \beta_{1}=\nu \wedge \bar{\nu}, \quad \beta_{2}=\nu \wedge \bar{\eta}, \quad \beta_{3}=\bar{\nu} \wedge \eta .
$$

Then

$$
\alpha \wedge \beta_{1}=d(-\theta \wedge \bar{\mu} \wedge \bar{\nu}), \quad \alpha \wedge \beta_{2}=d(-\theta \wedge \bar{\mu} \wedge \bar{\eta}), \quad \alpha \wedge \beta_{3}=d(\bar{\theta} \wedge \mu \wedge \eta) .
$$

All the forms $\alpha, \beta_{1}, \beta_{2}, \beta_{3}, \xi_{1}=-\theta \wedge \bar{\mu} \wedge \bar{\nu}, \xi_{2}=-\theta \wedge \bar{\mu} \wedge \bar{\eta}$ and $\xi_{3}=\bar{\theta} \wedge \mu \wedge \eta$ are $\mathbb{Z}_{3}$-invariant. Hence by (3.3) they descend to orbifold forms (denoted with a $\sim$ ) on the quotient $X=M / \mathbb{Z}_{3}$.

We consider the $a$-Massey product

$$
\left\langle a ; b_{1}, b_{2}, b_{3}\right\rangle
$$

for $a=[\tilde{\alpha}], b_{i}=\left[\tilde{\beta}_{i}\right] \in H^{2}(X), i=1,2,3$. By Nomizu's theorem mentioned earlier, the cohomology of $M$ up to degree 3 is

$$
\begin{aligned}
H^{0}(M, \mathbb{C})= & \langle 1\rangle, \\
H^{1}(M, \mathbb{C})= & \langle[\mu],[\bar{\mu}],[\nu],[\bar{\nu}],[\eta],[\bar{\eta}]\rangle, \\
H^{2}(M, \mathbb{C})= & \langle[\mu \wedge \bar{\mu}],[\mu \wedge \bar{\nu}],[\mu \wedge \theta],[\mu \wedge \eta],[\mu \wedge \bar{\eta}],[\bar{\mu} \wedge \nu],[\bar{\mu} \wedge \bar{\theta}],[\bar{\mu} \wedge \eta],[\bar{\mu} \wedge \bar{\eta}],[\nu \wedge \bar{\nu}], \\
& {[\nu \wedge \theta],[\nu \wedge \eta],[\nu \wedge \bar{\eta}],[\bar{\nu} \wedge \bar{\theta}],[\bar{\nu} \wedge \eta],[\bar{\nu} \wedge \bar{\eta}],[\bar{\eta} \wedge \bar{\eta}]\rangle, } \\
H^{3}(M, \mathbb{C})= & A \oplus \bar{A}
\end{aligned}
$$

where

$$
\begin{aligned}
A= & \langle[\mu \wedge \bar{\mu} \wedge \bar{\theta}],[\mu \wedge \bar{\mu} \wedge \eta],[\mu \wedge \nu \wedge \theta],[\mu \wedge \bar{\nu} \wedge \bar{\theta}],[\mu \wedge \bar{\nu} \wedge \eta],[\mu \wedge \theta \wedge \eta],[\mu \wedge \eta \wedge \bar{\eta}], \\
& {[\bar{\mu} \wedge \nu \wedge \bar{\theta}],[\bar{\mu} \wedge \nu \wedge \eta],[\bar{\mu} \wedge \bar{\theta} \wedge \eta],[\nu \wedge \bar{\nu} \wedge \bar{\theta}],[\nu \wedge \bar{\nu} \wedge \eta],[\nu \wedge \theta \wedge \eta],[\nu \wedge \eta \wedge \bar{\eta}], } \\
& {[\bar{\nu} \wedge \bar{\theta} \wedge \eta]\rangle . }
\end{aligned}
$$

Now $\mathbb{Z}_{3}$ acts on $A$ by multiplication with $\zeta$ and on $\bar{A}$ by multiplication with $\bar{\zeta}$, hence $H^{3}(X)=H^{3}(M)^{\mathbb{Z}_{3}}=0$. By [9, Proposition 2.7], the $a$-Massey product $\left\langle a ; b_{1}, b_{2}, b_{3}\right\rangle$ has no indeterminacy.

We denote by $q$ the projection $M \longrightarrow X$, and compute

$$
\begin{aligned}
\left\langle a ; b_{1}, b_{2}, b_{3}\right\rangle & =\left[\tilde{\xi}_{1} \wedge \tilde{\xi}_{2} \wedge \tilde{\beta}_{3}+\tilde{\xi}_{2} \wedge \tilde{\xi}_{3} \wedge \tilde{\beta}_{1}+\tilde{\xi}_{3} \wedge \tilde{\xi}_{1} \wedge \tilde{\beta}_{2}\right]= \\
& =q_{*}\left[\xi_{1} \wedge \xi_{2} \wedge \beta_{3}+\xi_{2} \wedge \xi_{3} \wedge \beta_{1}+\xi_{3} \wedge \xi_{1} \wedge \beta_{2}\right]= \\
& =2 q_{*}[\theta \wedge \mu \wedge \nu \wedge \eta \wedge \bar{\theta} \wedge \bar{\mu} \wedge \bar{\nu} \wedge \bar{\eta}]
\end{aligned}
$$


which is non-zero, since by (3.4) we have

$$
\begin{aligned}
\int_{X}\left\langle a ; b_{1}, b_{2}, b_{3}\right\rangle & =2 \int_{X} q_{*}[\theta \wedge \mu \wedge \nu \wedge \eta \wedge \bar{\theta} \wedge \bar{\mu} \wedge \bar{\nu} \wedge \bar{\eta}]= \\
& =6 \int_{M}[\theta \wedge \mu \wedge \nu \wedge \eta \wedge \bar{\theta} \wedge \bar{\mu} \wedge \bar{\nu} \wedge \bar{\eta}] \neq 0 .
\end{aligned}
$$

By Theorem 2.6 and Definition 3.8, the orbifold $X$ is non-formal.

\section{Simply CONNECTED SASAKian MANifOldS}

First, we recall some definitions and results on Sasakian manifolds (see [7] for more details).

An odd-dimensional Riemannian manifold $(N, g)$ is Sasakian if its cone $\left(N \times \mathbb{R}^{+}, g^{c}=\right.$ $\left.t^{2} g+d t^{2}\right)$ is Kähler, that is the cone metric $g^{c}=t^{2} g+d t^{2}$ admits a compatible integrable almost complex structure $J$ so that $\left(N \times \mathbb{R}^{+}, g^{c}=t^{2} g+d t^{2}, J\right)$ is a Kähler manifold. In this case the Reeb vector field $\xi=J \partial_{t}$ is a Killing vector field of unit length. The corresponding 1-form $\eta$ defined by $\eta(X)=g(\xi, X)$, for any vector field $X$ on $N$, is a contact form, meaning $\eta \wedge(d \eta)^{n} \neq 0$ at every point of $N$, where $\operatorname{dim} N=2 n+1$.

A Sasakian structure on $N$ is called quasi-regular if there is a positive integer $\delta$ satisfying the condition that each point of $N$ has a coordinate chart $(U, t)$ with respect to $\xi$ (the coordinate $t$ is in the direction of $\xi$ ) such that each leaf of $\xi$ passes through $U$ at most $\delta$ times. If $\delta=1$, then the Sasakian structure is called regular. (See [7, p. 188].) A result of [33] says that if $N$ admits a Sasakian structure, then it admits also a quasi-regular Sasakian structure.

If $M$ is a Kähler manifold whose Kähler form $\omega$ defines an integral cohomology class, then the total space of the circle bundle $S^{1} \hookrightarrow N \stackrel{\pi}{\longrightarrow} M$ with Euler class $[\omega] \in H^{2}(M, \mathbb{Z})$ is a regular Sasakian manifold with contact form $\eta$ such that $d \eta=\pi^{*}(\omega)$. The converse also holds: if $N$ is a regular Sasakian structure then the space of leaves $X$ is a Kähler manifold, and we have a circle bundle $S^{1} \hookrightarrow N \rightarrow M$ as above. If $N$ has a quasi-regular Sasakian structure, then the space of leaves $M$ is a Kähler orbifold with cyclic quotient singularities, and there is an orbifold circle bundle $S^{1} \hookrightarrow N \rightarrow X$ such that the contact form $\eta$ satisfies $d \eta=\pi^{*}(\omega)$, where $\omega$ is the orbifold Kähler form. Note that the map $\pi$ is an orbifold submersion, so that $\pi^{*}(\omega)$ is a well-defined (smooth) 2-form on the total space $N$, which is a smooth manifold. This defines a Sasakian structure on $N$ by [30, Theorem 20].

7.1. A simply connected non-formal Sasakian manifold. Examples of simply connected non-formal Sasakian manifolds, of dimension $2 n+1 \geq 7$, are given in [6]. There it is proved that those examples are non-formal because they are not 3-formal, in the sense of Definition 2.2. Here we show the non-formality proving that they have a non-trivial triple Massey product.

Note that if $N$ is a simply connected, compact and non-formal manifold (not necessarily Sasakian), then $\operatorname{dim} N \geq 7$. Indeed, Theorem 2.3 gives that simply connected compact manifolds of dimension at most 6 are formal [16, 31]. Moreover, a 7-dimensional simply connected Sasakian manifold is formal if and only if all the triple Massey products are trivial [29]. 
To construct a simply connected non-formal Sasakian 7-manifold, we consider the Kähler manifold $M=S^{2} \times S^{2} \times S^{2}$ with Kähler form

$$
\omega=\omega_{1}+\omega_{2}+\omega_{3},
$$

where $\omega_{1}, \omega_{2}$ and $\omega_{3}$ are the generators of the integral cohomology group of each of the $S^{2}$-factors on $S^{2} \times S^{2} \times S^{2}$. Let $N$ be the total space $N$ of the principal $S^{1}$-bundle

$$
S^{1} \hookrightarrow N \longrightarrow M=S^{2} \times S^{2} \times S^{2},
$$

with Euler class $[\omega] \in H^{2}(M, \mathbb{Z})$. Then, $N$ is a simply connected compact (regular) Sasakian manifold, with contact form $\eta$ such that $d \eta=\pi^{*}(\omega)$.

From now on, we write $a_{i}=\left[\omega_{i}\right] \in H^{2}\left(S^{2}\right)$. Since $M=S^{2} \times S^{2} \times S^{2}$ is formal, a model of $M$ is $\left(H^{*}\left(S^{2} \times S^{2} \times S^{2}\right), 0\right)$, where $H^{*}\left(S^{2} \times S^{2} \times S^{2}\right)$ is the de Rham cohomology algebra of $S^{2} \times S^{2} \times S^{2}$, that is

$$
\begin{aligned}
& H^{0}(M)=\langle 1\rangle, \\
& H^{1}(M)=H^{3}(M)=H^{5}(M)=0, \\
& H^{2}(M)=\left\langle a_{1}, a_{2}, a_{3}\right\rangle, \\
& H^{4}(M)=\left\langle a_{1} \cdot a_{2}, a_{1} \cdot a_{3}, a_{2} \cdot a_{3}\right\rangle, \\
& H^{6}(M)=\left\langle a_{1} \cdot a_{2} \cdot a_{3}\right\rangle .
\end{aligned}
$$

Therefore, a model of $N$ is the DGA $\left(H^{*}(M) \otimes \bigwedge(x), d\right)$, where $|x|=1, d\left(H^{*}(M)\right)=0$ and $d x=a_{1}+a_{2}+a_{3}$. By Lemma 2.4, we know that Massey products on a manifold can be computed by using any model for the manifold. Since $a_{1} \cdot a_{1}=0$ and $a_{1} \cdot a_{2}=\frac{1}{2} d\left(a_{1} \cdot x+\right.$ $\left.a_{2} \cdot x-a_{3} \cdot x\right)$, we have that the (triple) Massey product $\left\langle a_{1}, a_{1}, a_{2}\right\rangle=\frac{1}{2}\left[\left(a_{1} \cdot a_{2}-a_{1} \cdot a_{3}\right) \cdot x\right]$ is defined and it is non-trivial. Note that there is no indeterminacy of the Massey product, since it lives in $a_{1} \cdot H^{3}(N)+a_{2} \cdot H^{3}(N)$, but $H^{3}(N)=0$, since by the Gysin sequence, it equals the kernel of $[\omega]: H^{2}(M) \rightarrow H^{4}(M)$, which is an isomorphism. So $N$ is non-formal.

The case $n>3$ is similar and it is deduced as follows. Consider $B=S^{2} \times \stackrel{(n)}{.} \times S^{2}$. Let $a_{1}, \ldots, a_{n} \in H^{2}(B)$ be the cohomology classes given by each of the $S^{2}$-factors. Then the Kähler class is given by $[\omega]=a_{1}+\cdots+a_{n}$. Consider the circle bundle

$$
S^{1} \hookrightarrow N \longrightarrow B
$$

with first Chern class equal to $[\omega]$.

Using again Lemma 2.4, we know that Massey products on $N$ can be computed by using any model for $N$. Since $B$ is formal, a model of $B$ is the DGA $\left(H^{*}(B), 0\right)$. Thus, a model of $N$ is the DGA $\left(H^{*}(B) \otimes \bigwedge(x), d\right)$, where $|x|=1, d\left(H^{*}(B)\right)=0$ and $d x=a_{1}+a_{2}+\ldots+a_{n}$. Now, one can check that $a_{1} \cdot a_{1}=0$ and

$a_{1} \cdot a_{2} \ldots a_{n-2} \cdot a_{n-1}=\frac{1}{2} d\left(\left(a_{1} \cdot a_{2} \ldots a_{n-2}+a_{2} \cdot a_{3} \ldots a_{n-2} \cdot a_{n-1}-a_{2} \cdot a_{3} \ldots a_{n-2} \cdot a_{n}\right) \cdot x\right)$.

Thus the Massey product $\left\langle a_{1}, a_{1}, a_{2} \cdot a_{3} \ldots a_{n-2} \cdot a_{n-1}\right\rangle$ is defined and a representative is $\left[\left(a_{1} \cdot a_{2} \ldots a_{n-2} \cdot a_{n-1}-a_{1} \cdot a_{2} \ldots a_{n-2} \cdot a_{n}\right) \cdot x\right]$ which is non-trivial. Hence, we conclude that $N$ is non-formal. 
7.2. Simply connected formal Sasakian manifolds with $b_{2} \neq 0$. The most basic example of a simply connected compact regular Sasakian manifold is the odd-dimensional sphere $S^{2 n+1}$ considered as the total space of the Hopf fibration $S^{2 n+1} \hookrightarrow \mathbb{C P}^{n}$. It is well-known that $S^{2 n+1}$ is formal. In this section, we show examples of simply connected compact Sasakian manifolds, with second Betti number $b_{2} \neq 0$, which are formal.

Note that Theorem 2.3 implies that any simply connected compact manifold (Sasakian or not) of dimension $\leq 7$ and with $b_{2} \leq 1$, is formal. Examples of 7-dimensional simply connected compact Sasakian manifolds, with $b_{2} \geq 2$, which are formal are given in [15].

To show examples of simply connected formal Sasakian manifolds, of dimension $\geq 9$ and with $b_{2} \neq 0$, we consider the Kähler manifold

$$
M=\mathbb{C P}^{n-1} \times S^{2},
$$

with Kähler form

$$
\omega=\omega_{1}+\omega_{2},
$$

where $\omega_{1}$ and $\omega_{2}$ are the generators of the integral cohomology group of $\mathbb{C P}^{n-1}$ and $S^{2}$, respectively. Let $N$ be the total space $N$ of the principal $S^{1}$-bundle

$$
S^{1} \hookrightarrow N \longrightarrow M=\mathbb{C P}^{n-1} \times S^{2},
$$

with Euler class $[\omega] \in H^{2}(M, \mathbb{Z})$. Then, $N$ is a simply connected compact (regular) Sasakian manifold, of dimension $2 n+1$, with contact form $\eta$ such that $d \eta=\pi^{*}(\omega)$.

Proposition 7.1. The total space $N$ of the circle bundle $S^{1} \hookrightarrow N \longrightarrow M=\mathbb{C P}^{n-1} \times$ $S^{2}$, with Euler class $[\omega]$, is a simply connected compact Sasakian manifold, with second Betti number $b_{2}=1$, which is formal.

Proof. Suppose $n \geq 4$. We will determine a minimal model of the $(2 n+1)$-manifold $N$.

Clearly $M=\mathbb{C P}^{n-1} \times S^{2}$ is formal because $M$ is Kähler. Hence, a (non-minimal) model of $M$ is the DGA $\left(H^{*}(M), 0\right)$, where $H^{*}(M)$ is the de Rham cohomology algebra of $M$. Thus, a (non-minimal) model of $N$ is the differential algebra $(\mathcal{A}, d)$, where

$$
\mathcal{A}=H^{*}(M) \otimes \bigwedge(x), \quad|x|=1, \quad d\left(H^{*}(M)\right)=0, \quad d x=a_{1}+a_{2},
$$

where $a_{1}$ is the integral cohomology class defined by the Kähler form $\omega_{1}$ on $\mathbb{C P}^{n-1}$, and $a_{2}$ is the integral cohomology class defined by the Kähler form $\omega_{2}$ on $S^{2}$. Then, the minimal model associated to this model of $N$ is

$$
(\mathcal{M}, D)=(\bigwedge(a, b, z), D)
$$

where $|a|=2,|b|=3$ and $|z|=2 n-1$, while the differential $D$ is given by $D a=D b=$ 0 and $D z=a^{n}$. Therefore, we get

$$
N^{i}=0,
$$

for $1 \leq i \leq n$. Then, Theorem 2.3 implies that $N$ is formal because it is $n$-formal.

7.3. Non-formal quasi-regular Sasakian manifolds with $b_{1}=0$. The previous examples can be tweaked to obtain also examples of quasi-regular Sasakian manifolds $P$, where the base of the (orbifold) circle bundle $S^{1} \hookrightarrow P \rightarrow X$ is an honest orbifold Kähler manifold $X$. Obtaining simply connected manifolds $P$ in this way is a delicate matter, since the fundamental group of $P$ relates to the orbifold fundamental group of $X$, and not 
its fundamental group (see [25] and [30] for discussions on these issues). Therefore we content ourselves with writing down examples with $H_{1}(P, \mathbb{Z})=0$.

Consider a complex 3-torus $T^{3}=\mathbb{C}^{3} / \Gamma$, where $\Gamma$ is the discrete subgroup of $\mathbb{C}^{3}$ consisting of the elements $\left(z_{1}, z_{2}, z_{3}\right) \in \mathbb{C}^{3}$ whose components $z_{1}, z_{2}$ and $z_{3}$ are Gaussian integers. Now consider the action of the finite group $\mathbb{Z}_{2}$ on $\mathbb{C}^{3}$ given by

$$
\begin{aligned}
\varphi: \mathbb{C}^{3} & \rightarrow \mathbb{C}^{3} \\
\left(z_{1}, z_{2}, z_{3}\right) & \mapsto\left(-z_{1},-z_{2},-z_{3}\right),
\end{aligned}
$$

where $\varphi$ is the generator of $\mathbb{Z}_{2}$. This action satisfies that $\varphi\left(z+z^{\prime}\right)=\varphi(z)+\varphi\left(z^{\prime}\right)$, for $z, z^{\prime} \in \mathbb{C}^{3}$. Moreover, $\varphi(\Gamma)=\Gamma$. Therefore, $\varphi$ induces an action on $T^{3}=\mathbb{C}^{3} / \Gamma$ with $2^{6}$ fixed points corresponding to $\left(z_{1}=u_{1}+i u_{2}, z_{2}=u_{3}+i u_{4}, z_{3}=u_{5}+i u_{6}\right)$ with $u_{i}=0, \frac{1}{2}$. Thus, the quotient space

$$
X=T^{3} / \mathbb{Z}_{2}
$$

is a Kähler orbifold of (real) dimension 6 with $2^{6}$ isolated orbifold singularities of order 2 . In fact, one can check that the standard complex structure $J$ on $T^{3}$ commutes with the $\mathbb{Z}_{2}$-action, that is $\left(\varphi_{*}\right)_{z} \circ J_{z}=J_{\varphi(z)} \circ\left(\varphi_{*}\right)_{z}$, for any point $z \in T^{3}$. Moreover, the standard Hermitian metric and the Kähler form $\omega^{\prime}$ on $T^{3}$ are $\mathbb{Z}_{2}$-invariant, and so they induce an orbifold Hermitian metric and an orbifold Kähler form $\omega$ on $X$, respectively.

By (3.3), the cohomology of $X$ is given by $H^{1}(X, \mathbb{Z})=H^{1}\left(T^{3}, \mathbb{Z}\right)^{\mathbb{Z}_{2}}=0$, hence $b_{1}(X)=$ 0 . Now consider the orbifold circle bundle

$$
S^{1} \hookrightarrow P \stackrel{\pi}{\longrightarrow} X,
$$

given by $c_{1}(P)=[\omega]$. We have the following:

Proposition 7.2. The manifold $P$ is a 7-dimensional quasi-regular Sasakian manifold $N$ with $b_{1}=0$ which is non-formal.

Proof. The total space of the orbifold circle bundle $P$ has a Sasakian structure with contact form $\eta$ such that $d \eta=\pi^{*}(\omega)$, by [30, Theorem 20] (the proof of this result is given in the K-contact case but it works also for the Sasakian case). The Leray spectral sequence gives that $b_{1}(P)=0$.

Let us see that $P$ is non-formal. First note that the cohomology of $T^{3}$ is the exterior algebra $\bigwedge^{*}\left(x_{1}, \ldots, x_{6}\right)$, with $\left|x_{i}\right|=1,1 \leq i \leq 6$. Then $H^{*}(X)=\bigwedge^{\text {even }}\left(x_{1}, \ldots, x_{6}\right)$. Let $a_{1}=x_{1} x_{2}, a_{2}=x_{3} x_{4}, a_{3}=x_{5} x_{6}$, so that $[\omega]=a_{1}+a_{2}+a_{3}$. As in Subsection [7.1, there is non-trivial (triple) Massey product in $P$. Indeed, $a_{1} \cdot a_{1}=0$ and $a_{1} \cdot a_{2}=$ $\frac{1}{2} d\left(\left(a_{1}+a_{2}-a_{3}\right) \cdot \eta\right)$. Then,

$$
\left\langle a_{1}, a_{1}, a_{2}\right\rangle=\frac{1}{2}\left[\left(a_{1} \cdot a_{2}-a_{1} \cdot a_{3}\right) \cdot \eta\right]
$$

where $d \eta=\pi^{*}(\omega)$. So $P$ is non-formal.

There is a geometrical explanation of the above Massey product. If $T=\mathbb{C} / \mathbb{Z}^{2}$ is the 2-torus, then the quotient $T / \mathbb{Z}_{2} \cong S^{2}$, as a topological manifold. Thus

$$
T^{3} /\left(\mathbb{Z}_{2} \times \mathbb{Z}_{2} \times \mathbb{Z}_{2}\right)=\left(T / \mathbb{Z}_{2}\right) \times\left(T / \mathbb{Z}_{2}\right) \times\left(T / \mathbb{Z}_{2}\right) \cong S^{2} \times S^{2} \times S^{2}=M,
$$

where each of the factors of $\mathbb{Z}_{2} \times \mathbb{Z}_{2} \times \mathbb{Z}_{2}$ acts on each of the three factors of $T^{3}=T \times T \times T$, respectively, and $M$ is the 6 -manifold of Subsection 7.1. Therefore, the orbifold $X$ sits in 
the middle of two quotient maps

$$
T^{3} \rightarrow X=T^{3} / \mathbb{Z}_{2} \rightarrow M \cong T^{3} /\left(\mathbb{Z}_{2} \times \mathbb{Z}_{2} \times \mathbb{Z}_{2}\right) .
$$

Then there is a diagram

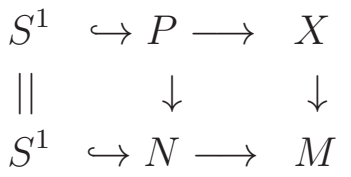

where $N$ is the 7-manifold of Subsection 7.1. So, $P$ and $N$ are the same topological manifold. Then the non-zero Massey product of $N$ produces the non-zero Massey product for $P$, giving the non-formality of $P$.

\section{ACKNOWLEDGEMENTS}

We are grateful to the referees for their helpful comments. The first author is supported by a Post-Doc grant at Philipps-Universität Marburg. The second author is supported by the J. C. Bose Fellowship. The third author is partially supported through Project MINECO (Spain) MTM2014-54804-P and Basque Government Project IT1094-16. The fourth author is partially supported by Project MINECO (Spain) MTM2015-63612-P.

\section{REFERENCES}

[1] A. Adem, J. Leida And Y. Ruan, Orbifolds and string theory, Cambridge Univ. Press, 2007.

[2] M. АтіYAH, Elliptic operators and compact groups, Lecture Notes in Math. 401, Springer-Verlag, 1974.

[3] W. Baily, The decomposition theorem for $V$-manifolds, Amer. J. Math. 78 (1956), 862-888.

[4] W. BAILY, On the imbedding of $V$-manifolds in projective space, Amer. J. Math. 79 (1957), 403-430.

[5] G. Bazzoni, M. Fernández and V. Muñoz, A 6-dimensional simply connected complex and symplectic manifold with no Kähler metric, to appear in J. Symplectic Geom. arXiv:1410.6045.

[6] I. Biswas, M. Fernández, V. Muñoz And A. Tralle, On formality of Sasakian manifolds, J. Topol. 9 (2016), 161-180.

[7] C. Boyer And K. Galicki, Sasakian Geometry, Oxford Univ. Press, Oxford, 2007.

[8] G. E. Bredon, Introduction to Compact Transformation Groups, Pure and Applied Mathematics 46, Academic Press, 1972.

[9] G. Cavalcanti, M. Fernández and V. Muñoz, Symplectic resolutions, Lefschetz property and formality, Adv. Math. 218 (2008), 576-599.

[10] L. Cordero And R. Wolak, Properties of the basic cohomology of transversely Kähler foliations, Rend. Circolo Mat. Palermo 40 (1991), 177-188.

[11] P. Deligne, P. Griffiths, J. Morgan and D. Sullivan, Real homotopy theory of Kähler manifolds, Invent. Math. 29 (1975), 245-274.

[12] L.J. Dixon, J.A. Harvey, C. VAfa And E. Witten, Strings on orbifolds I, Nuclear Phys. B 261 (1985), 678-686.

[13] A. El Kacimi Alaoui, Opérateurs transversalement elliptiques sur un feuilletage riemannien et applications, Compositio Math. 73 (1990), 57-106.

[14] Y. Felix, S. Halperin and J.-C. Thomas, Rational Homotopy Theory, Springer, 2002.

[15] M. Fernández, S. Ivanov And V. Muñoz, Formality of 7-dimensional 3-Sasakian manifolds, arXiv:1511.08930.

[16] M. Fernández And V. Muñoz, Formality of Donaldson submanifolds, Math. Z. 250 (2005), 149175 .

[17] M. Fernández AND V. MuÑoz, An 8-dimensional non-formal simply connected symplectic manifold, Annals of Math. 167 (2008), 1045-1054. 
[18] J. Girbau, A. Haefliger and D. Sundararaman, On deformations of transversely holomorphic foliations, J. Reine Angew Math. 345 (1983), 122-147.

[19] P. Griffiths And J. Morgan, Rational homotopy theory and differential forms, Progress in Math. 16, Birkhäuser, 1981.

[20] S. Halperin, Lectures on minimal models, Mém. Soc. Math. France 230, 1983.

[21] T. KAWASAKI, The signature theorem for $V$-manifolds, Topology 17 (1978), 75-83.

[22] T. KaWASAKI, The Riemann-Roch theorem for complex V-manifolds, Osaka J. Math. 16 (1979), 151-159.

[23] T. KAwASAKI, The index of elliptic operators over V-manifolds, Nagoya Math. J. 84 (1981), 135157.

[24] B. Kleiner and J. Lott, Geometrization of three-dimensional orbifolds via Ricci flow, Astérisque 365 (2014), 101-177.

[25] J. Kollár, Shafarevich maps and plurigenera of algebraic varieties, Inventiones Math. 113 (1993), $177-216$.

[26] J. Kollár, Circle actions on simply connected 5-manifolds, Topology 45(2006), 643-672

[27] I. MoerdiJK AND J. MrCun, Introduction to foliations and Lie groupoids, Cambridge Studies in Adv. Math. 91, Cambridge Univ. Press, 2003.

[28] I. Moerdijk and D. A. Pronk, Orbifolds, sheaves and groupoids, K-Theory 12 (1997), 3-21.

[29] V. Muñoz And A. Tralle, Simply connected K-contact and Sasakian manifolds of dimension 7, Math. Z. 281 (2015), 457-470.

[30] V. Muñoz, J. A. Rojo And A. Tralle, Homology Smale-Barden manifolds with K-contact and Sasakian structures, arXiv:1601.06136

[31] J. Neisendorfer And T. Miller, Formal and coformal spaces, Illinois. J. Math. 22 (1978), $565-580$.

[32] K. Nomizu, On the cohomology of compact homogeneous spaces of nilpotent Lie groups, Annals of Math. 59 (1954), 531-538.

[33] L. Ornea And M. Verbitsky, Sasakian structures on CR-manifolds, Geom. Dedicata 125 (2007), 159-173.

[34] M. Pflaum, Analytic and geometric study of stratified spaces, Lecture Notes in Math. 1768, Springer, 2001.

[35] I. SatAke, On a generalization of the notion of manifold, Proc. Nat. Acad. Sci. USA 42 (1956), 359-363.

[36] I. Satake, The Gauss-Bonnet theorem for V-manifolds, J. Math. Soc. Japan 9 (1957), 464-492.

[37] D. Sullivan, Infinitesimal computations in topology, Inst. Hautes Études Sci. Publ. Math. 47 (1978), 269-331.

[38] W. P. Thurston, The geometry and topology of 3-manifolds, Mimeographed Notes, Princeton University, 1979.

[39] P. Tondeur, Geometry of foliations, Monographs in Math. 90, Birkhäuser Verlag, 1997.

[40] A. Tralle And J. OpreA, Symplectic manifolds with no Kähler structure, Lecture Notes in Math. 1661, Springer-Verlag, 1997.

[41] M. Verbitsky, Hodge theory on nearly Kähler manifolds, Geom. Topol. 15 (2011), 2111-2133.

[42] Z. Wang And D. Zaffran, A remark on the Hard Lefschetz theorem for Kähler orbifolds, Proc. Amer. Math. Soc. 137 (2009), 2497-2501.

[43] R. Wells, Differential analysis on complex manifolds, Graduate Texts in Mathematics, 65, SpringerVerlag, New York-Berlin, 1980. 
Philipps-Universität Marburg, FB Mathematik \& Informatik, Hans-Meerwein-Str. 6 Campus Lahnberge, 35032 Marburg, Germany

E-mail address: bazzoni@mathematik.uni-marburg.de

School of Mathematics, Tata Institute of Fundamental Research, Homi Bhabha Road, BOMBAY 400005, INDIA

E-mail address: indranil@math.tifr.res.in

Universidad del País Vasco, Facultad de Ciencia y Tecnología, Departamento de Matemáticas, Apartado 644, 48080 Bilbao, Spain

E-mail address: marisa.fernandez@ehu.es

Facultad de Ciencias Matemáticas, Universidad Complutense de Madrid, Plaza de Ciencias 3, 28040 Madrid, Spain

E-mail address: vicente.munoz@mat.ucm.es

Department of Mathematics and Computer Science, University of Warmia and Mazury, SŁoneczna 54, 10-710, Olsztyn, Poland

E-mail address: tralle@matman.uwm.edu.pl 